\title{
MULTILINEAR HANKEL FORMS OF HIGHER ORDER AND ORTHOGONAL POLYNOMIALS
}

\author{
HJALMAR ROSENGREN
}

\begin{abstract}
.
The theory of bilinear Hankel forms of higher order (or higher weight) initiated in [12] is extended to the multilinear case. We study multilinear forms appearing in the decomposition of tensor products of function spaces on the disc with respect to a weighted action of the Möbius group. We obtain a trace ideal criterion for these forms, and study discrete and continuous systems of orthogonal polynomials in several variables connected with the decomposition. Some of these polynomials are related to spherical harmonics.
\end{abstract}

\section{Introduction.}

Let $\mathbf{D}$ be the unit disc in C. We will denote by $G$ the Möbius group of conformal automorphisms of $\mathbf{D}$ and by $\tilde{G}$ its universal cover. One can define an element of $\tilde{G}$ to be an element $\phi$ of $G$ and a determination of $\log \phi^{\prime}$. We will abusively denote such an element by the same letter $\phi$. Then, for any $\nu \in \mathrm{C}$,

$$
(f * \phi)(z)=f(\phi z) \phi^{\prime}(z)^{\nu / 2}
$$

defines an action of $\tilde{G}$ on functions on $\mathbf{D}$. When $\nu$ is a real integer we have an action of the double cover $\mathrm{SU}(1,1) \simeq \mathrm{SL}(2, \mathrm{R})$ of $G$, and if $\nu$ is even, an action of $G$. We will be concerned with the case when $\nu>0$ and the functions $f$ are analytic. There is then an essentially unique [4] Hilbert space $\mathscr{A}^{\nu}$ of analytic functions on the disc whose norm is invariant under the action (1), so that

$$
U_{\nu}(\phi) f=f * \phi^{-1}
$$

gives a unitary representation of $\tilde{G}$ on $\mathscr{A}^{\nu}$. The unitary representations of $\tilde{G}$ were classified in [26]. What we have here is the positive discrete series, in a realization which was first considered in this generality in [28]. The scalar product in the space $\mathscr{A}^{\nu}$ is given by

Received November 27, 1995. 


$$
\langle f, g\rangle_{\nu}=\sum_{k=0}^{\infty} \frac{k !}{(\nu)_{k}} \hat{f}(k) \overline{\hat{g}(k)}
$$

where $f(z)=\sum \hat{f}(k) z^{k}$ and

$$
(\nu)_{k}=\nu(\nu+1) \cdots(\nu+k-1), \quad(\nu)_{0}=1
$$

is the Pochhammer symbol. The norm will be denoted by \|\|$_{\nu}$, it is normalized so that $\|1\|_{\nu}=1$. The space $\mathscr{A}^{\nu}$ has the reproducing kernel $k_{w}(z)=(1-\bar{w} z)^{-\nu}$, that is

$$
f(w)=\left\langle f, k_{w}\right\rangle_{\nu}, \quad f \in \mathscr{A}^{\nu}, w \in \mathbf{D} .
$$

When $\nu>1, \mathscr{A}^{\nu}$ is the Dzhrbashyan (or weighted Bergman) space [9], with inner product

$$
\langle f, g\rangle_{\nu}=\int_{\mathbf{D}} f(z) \overline{g(z)} d m_{\nu}(z)
$$

where

$$
d m_{\nu}(z)=\frac{\nu-1}{\pi}\left(1-|z|^{2}\right)^{\nu-2} d x d y .
$$

In particular, $\mathscr{A}^{2}$ is the (unweighted) Bergman space. When $\nu=1$, we have the Hardy space $\mathrm{H}^{2}$ with inner product

$$
\langle f, g\rangle_{1}=\frac{1}{2 \pi} \int_{\partial \mathbf{D}} f \bar{g}|d z| .
$$

For $0<\nu<1, \mathscr{A}^{\nu}$ is not the analytic part of an $L^{2}$-space, so there is no so simple integral formula. We are interested in bounded multilinear forms on a product $\mathscr{A}^{\nu_{1}} \times \cdots \times \mathscr{A}^{\nu_{n}}$. These can uniquely be written as

$$
\left(f_{1}, \ldots, f_{n}\right) \mapsto \sum_{k_{1}, \ldots, k_{n}=0}^{\infty} \frac{k_{1} ! \cdots k_{n} !}{\left(\nu_{1}\right)_{k_{1}} \cdots\left(\nu_{n}\right)_{k_{n}}} \hat{f}_{1}\left(k_{1}\right) \cdots \hat{f}_{n}\left(k_{n}\right) \overline{\hat{K}\left(k_{1}, \ldots, k_{n}\right)},
$$

where the kernel $K(\mathbf{z})=\sum \hat{K}\left(k_{1}, \ldots, k_{n}\right) \mathbf{z}^{k}$ is analytic on the polydisc $\mathbf{D}^{n}$; here and throughout we use multi-index notation such as

$$
\mathbf{z}=\left(z_{1}, \ldots, z_{n}\right), \quad \mathbf{z}^{k}=z_{1}^{k_{1}} \cdots z_{n}^{k_{n}} .
$$

We have an action of $\tilde{G}$, which on the kernel level takes the form

$$
(K * \phi)(\mathbf{z})=K\left(\phi z_{1}, \ldots, \phi z_{n}\right) \phi^{\prime}\left(z_{1}\right)^{\frac{1}{2} \nu_{1}} \cdots \phi^{\prime}\left(z_{n}\right)^{\frac{1}{2} \nu_{n}} .
$$

The Hilbert tensor product $\mathscr{A}^{\nu_{1}} \otimes \cdots \otimes \mathscr{A}^{\nu_{n}}$ can be identified with the space of kernels such that 


$$
\|K\|^{2}=\sum_{k_{1}, \ldots, k_{n}=0}^{\infty} \frac{k_{1} ! \cdots k_{n} !}{\left(\nu_{1}\right)_{k_{1}} \cdots\left(\nu_{n}\right)_{k_{n}}}\left|\hat{K}\left(k_{1}, \ldots, k_{n}\right)\right|^{2}<\infty,
$$

or with the corresponding forms. $\tilde{G}$ acts unitarily on this Hilbert space by

$$
\left(U_{\nu_{1}} \otimes \cdots \otimes U_{\nu_{n}}\right)(\phi) K=K * \phi^{-1} .
$$

When $\nu=\left(\nu_{1}, \ldots, \nu_{n}\right)$ is fixed we will use the notations $\langle,\rangle_{\nu},\|\|_{\nu}$ for this space as well, and denote it for short by $\otimes \mathscr{A}^{\nu}$. When $\nu_{i}>1$, we will denote the measure $d m_{\nu_{1}}\left(z_{1}\right) \cdots d m_{\nu_{n}}\left(z_{n}\right)$ by $d m_{\nu}(\mathbf{z})$, so that $\otimes \mathscr{A}^{\nu}$ is the analytic part of $L^{2}\left(\mathbf{D}^{n}, d m_{\nu}\right)$.

Note that although $\mathscr{A}^{\nu}$ is irreducible under the action of $\tilde{G}$, the tensor product is not. In fact, there is a decomposition

$$
\mathscr{A}^{\nu_{1}} \otimes \mathscr{A}^{\nu_{2}}=\bigoplus_{s=0}^{\infty} \mathscr{H}_{s}
$$

into closed irreducible subspaces. When viewed as bilinear forms, the elements of $\mathscr{H}_{s}$ can be written as

$$
H\left(f_{1}, f_{2}\right)=H_{g}^{s}\left(f_{1}, f_{2}\right)=\left\langle\mathscr{J}_{s}\left(f_{1}, f_{2}\right), g\right\rangle_{\mu},
$$

where $\mu=\nu_{1}+\nu_{2}+2 s$, the symbol $g$ is in $\mathscr{A}^{\mu}$, and $\mathscr{J}_{s}$ is given by

$$
\mathscr{J}_{s}\left(f_{1}, f_{2}\right)=\sum_{k=0}^{s}\left(\begin{array}{l}
s \\
k
\end{array}\right)(-1)^{s-k} \frac{f_{1}^{(k)} f_{2}^{(s-k)}}{\left(\nu_{1}\right)_{k}\left(\nu_{2}\right)_{s-k}} .
$$

This is shown in [12] for $\nu_{1}=\nu_{2}$ and in [33] for $\nu_{1} \neq \nu_{2}$. The bilinear differential operators $\mathscr{J}_{s}$ are called transvectants. (To obtain the transvectants occuring in classical invariant theory, one formally replaces the $\nu_{i}$ with $n e-$ gative integers. For higher order Hankel forms in this context, cf. [22].) In particular, $\mathscr{H}_{0}$ is the space of forms

$$
\left(f_{1}, f_{2}\right) \mapsto\left\langle f_{1} f_{2}, g\right\rangle_{\nu_{1}+\nu_{2}} .
$$

This is usually called a Hankel form, and accordingly the elements of $\mathscr{H}_{s}$ for general $s$ are called Hankel forms of higher order (or higher weight).

A fundamental fact is that the correspondence between $H$ and $g$ gives an equivalence of unitary representations, that is

$$
\left\|H_{g}^{s}\right\|_{\nu}=C\|g\|_{\mu}, \quad\left(U_{\nu_{1}} \otimes U_{\nu_{2}}\right)(\phi) H_{g}^{s}=H_{U_{\mu}(\phi) g}^{s}
$$

(where $C$ is a constant depending on $s, \nu_{1}$ and $\nu_{2}$ ), so that

$$
\mathscr{A}^{\nu_{1}} \otimes \mathscr{A}^{\nu_{2}} \simeq \bigoplus_{s=0}^{\infty} \mathscr{A}^{\nu_{1}+\nu_{2}+2 s}
$$


In itself, this equivalence is not very hard to prove. However, in the papers mentioned it is given a very explicit form. Moreover, the operators $H_{g}^{s}$ make sense also for more general symbols $g$, and one still has the intertwining property $\left(H_{g}^{s}\right) * \phi=H_{g * \phi}^{s}$. This arises for example the question of relating Schatten class properties of these forms to properties of the symbol the Hilbert tensor product equals the Schatten class $\mathfrak{\Xi}_{2}$ ), which is answered in $[12,33]$ (for $\nu_{1}, \nu_{2} \geq 1$ ).

In this paper we study the general case $\mathscr{A}^{\nu_{1}} \otimes \cdots \otimes \mathscr{A}^{\nu_{n}}$. From (7) follows by induction the equivalence

$$
\mathscr{A}^{\nu_{1}} \otimes \cdots \otimes \mathscr{A}^{\nu_{n}} \simeq \bigoplus_{s_{1}, \ldots, s_{n-1}=0}^{\infty} \mathscr{A}^{|\nu|+2\left(s_{1}+\cdots+s_{n-1}\right)}=\bigoplus_{s=0}^{\infty}\left(\begin{array}{c}
n+s-2 \\
n-2
\end{array}\right) \mathscr{A}^{|\nu|+2 s}
$$

(where $|\nu|=\sum \nu_{i}$ ), which we intend to make more explicit. In Section 2 we start our study of this decomposition by considering the derived representation of the Lie algebra of $\tilde{G}$. In Section 3 we give explicit expressions for the elements of the components, involving multilinear generalizations of the transvectant and Newton's divided differences. In Section 4 we study Schatten class properties of our forms. Our approach is different from that in [12]. The proof of that paper depends on the theory of paracommutators, whereas we use the theory of Möbius invariant function spaces. In Sections 5 to 7 we generalize some of the contents of [19]. That is we study certain discrete and continuous systems of orthogonal polynomials connected with the decomposition, and more precisely with the Clebsch-Gordan and Wigner coefficients of our representation. In the bilinear case three classical polynomial systems appear, namely the Jacobi polynomials, the Hahn polynomials and the Hahn polynomials of imaginary argument. In the $n$-linear case we obtain analogues of these with $n-1$ variables. Special cases of these polynomials have been introduced by Appell [1], Karlin and McGregor [13] and Proriol [25]. When the $\nu_{i}$ are half-integers, the polynomials of Jacobi-type are related to spherical harmonics. This is explained in Section 8. In fact we sketch an alternative approach to higher order Hankel forms, based on the SegalShale-Weil representation, where harmonic polynomials arise in a natural way.

We would like to point out that the disc is just the first case to consider. It should be possible to extend our findings to more general domains. In the bilinear case, this has been studied in [20] for the complex ball and for general bounded symmetric domains in [21]. Another generalization involves $q$ analogues of the spaces $\mathscr{A}^{\nu}$, where the Lie group $\tilde{G}$ is replaced by a "quantum group". This gives rise to basic hypergeometric polynomials of several variables. We will treat this in detail in the future publication [27]. For further ideas of generalizations we refer to [23]. 
ACKNowledgement. I am most grateful to Jaak Peetre for suggesting me to study this topic, and for his invaluable advice while I was working with this paper. I also thank Genkai Zhang for fruitful discussions; the alternative approach of Section 8 is based on his suggestions.

\section{An infinitesimal approach.}

In this section we apply some fundamental facts about highest weight modules to the space $\otimes \mathscr{A}^{\nu}=\mathscr{A}^{\nu_{1}} \otimes \cdots \otimes \mathscr{A}^{\nu_{n}}$. Recall the derived representation, which is defined by

$$
X f=\lim _{t \rightarrow 0} \frac{U_{\nu}(\exp (t X)) f-f}{t}
$$

for $X$ in the Lie algebra $\mathfrak{s u}(1,1)$ of $\tilde{G}$. (Compared to [12], we have replaced $X f$ by $-X f$. This is one reason why we prefer to speak of forms of higher order, since strictly speaking these are now of lower weight.) It extends by linearity to the complexification $\mathfrak{g}=\mathfrak{s l}(2, \mathrm{C})$ of $\mathfrak{s u}(1,1)$, which is more convenient to use. In the standard basis $E=\left(\begin{array}{ll}0 & 1 \\ 0 & 0\end{array}\right), F=\left(\begin{array}{ll}0 & 0 \\ 1 & 0\end{array}\right), H=\left(\begin{array}{cc}1 & 0 \\ 0 & -1\end{array}\right)$ of $\mathfrak{g}$ it is given on $\mathscr{A}^{\nu}$ by

$$
E=-\frac{d}{d z}, \quad F=z^{2} \frac{d}{d z}+\nu z, \quad H=-\left(2 z \frac{d}{d z}+\nu\right),
$$

and on $\otimes \mathscr{A}^{\nu}$ by

$$
E=-\sum_{i=1}^{n} \frac{\partial}{\partial z_{i}}, \quad F=\sum_{i=1}^{n}\left(z_{i}^{2} \frac{\partial}{\partial z_{i}}+\nu_{i} z_{i}\right), \quad H=-\sum_{i=1}^{n}\left(2 z_{i} \frac{\partial}{\partial z_{i}}+\nu_{i}\right) .
$$

When considered as operators, they are unbounded, though densely defined. They satisfy

$$
\begin{gathered}
{[E F]=H, \quad[H E]=2 E, \quad[H F]=-2 F,} \\
E^{*}=-F, \quad F^{*}=-E, \quad H^{*}=H,
\end{gathered}
$$

where $*$ denotes the Hilbert space adjoint. We also compute the Casimir operator

$$
\begin{aligned}
C & =H^{2}+2 E F+2 F E=\sum_{i} \nu_{i}\left(\nu_{i}-2\right) \\
& +2 \sum_{i<j}\left(\nu_{i} \nu_{j}+2\left(z_{i}-z_{j}\right)\left(\nu_{j} \frac{\partial}{\partial z_{i}}-\nu_{i} \frac{\partial}{\partial z_{j}}\right)-2\left(z_{i}-z_{j}\right)^{2} \frac{\partial^{2}}{\partial z_{i} \partial z_{j}}\right) .
\end{aligned}
$$

This is a symmetric operator which commutes with the actions of $\mathfrak{g}$ and $\tilde{G}$.

An irreducible $\mathfrak{g}$-module of highest weight $\lambda$ is by definition a module $A$ 
generated by a vector of highest weight $\lambda$, i.e. an element $q \neq 0$ satisfying $E q=0, H q=\lambda q$. Then

$$
E F^{k} q=k(\lambda-k+1) F^{k-1} q, \quad H F^{k} q=(\lambda-2 k) F^{k} q,
$$

so $A$ is generated over $\mathrm{C}$ by $\left\{F^{k} q \mid k=0,1,2,\right\}$. Also $C p=\lambda(\lambda+2) p$ for all $p \in A$.

Let us for a while confine attention to the space $\mathscr{P}_{i}$ of polynomials, considered as a $\mathfrak{g}$-submodule of $\mathscr{A}^{\nu_{i}}$. It is an irreducible module of highest weight $-\nu_{i}$, with 1 as a highest weight vector. By purely algebraic arguments (cf. [11], p. 71) it follows that

$$
\bigotimes_{i=1}^{n} \mathscr{P}_{i}=\bigoplus_{s=0}^{\infty} H_{s}
$$

where $H_{s}$ is a sum of $\left(\begin{array}{c}n+s-2 \\ n-2\end{array}\right)$ irreducible $\mathfrak{g}$-modules of highest weight $-(|\nu|+2 s)$. Writing $\mathscr{H}_{s}$ for the closure of $H_{s}$ in $\bigotimes \mathscr{A}^{\nu}$, it follows that

$$
\bigotimes_{i=1}^{n} \mathscr{A}^{\nu_{i}}=\bigoplus_{s=0}^{\infty} \mathscr{H}_{s} \simeq \bigoplus_{s=0}^{\infty}\left(\begin{array}{c}
n+s-2 \\
n-2
\end{array}\right) \mathscr{A}^{|\nu|+2 s},
$$

where the sum and tensor product now are interpreted in the Hilbert space sense. Note that $\mathscr{H}_{s}$ is also the eigenspace of $C$ with the eigenvalue $(|\nu|+2 s)(|\nu|+2 s-2)$. We define a Hankel form of higher order to be a multilinear form of type (4) whose kernel $K$ is a eigenvector (not necessarily in $\otimes \mathscr{A}^{\nu}$ ) of $C$. A Hankel form of order $s$ is a form with the eigenvalue $(|\nu|+2 s)(|\nu|+2 s-2)$.

\section{Explicit expressions.}

In this section we find some explicit expressions for our generalized Hankel forms. Let us first determine the highest weight vectors, i.e. the solutions in $\otimes \mathscr{A}^{\nu}=\mathscr{A}^{\nu_{1}} \otimes \cdots \otimes \mathscr{A}^{\nu_{n}}$ to the system $H q=-(|\nu|+2 s) q, E q=0$. The first equation means that they are homogeneous polynomials of degree $s$, the second one that they can be expressed as functions of $z_{i}-z_{j}(1 \leq i, j \leq n)$. Alternatively, the second equation means that

$$
q\left(z_{1}+w, \ldots, z_{n}+w\right)=q\left(z_{1}, \ldots, z_{n}\right)
$$

for all $w$. The space of all such polynomials has indeed dimension $\left(\begin{array}{c}n+s-2 \\ n-2\end{array}\right)$.

We now fix such a highest weight polynomial $q$. It will be convenient to write

$$
\mu=|\nu|+2 s .
$$


We want to construct the intertwining embedding $\mathscr{K}_{q}$ from $\mathscr{A}^{\mu}$ to $\otimes \mathscr{A}^{\nu}$ that takes 1 to $q$. Then $w^{k}=F^{k} 1 /(\mu)_{k}$ should be mapped to $F^{k} q /(\mu)_{k}$, so we set

$$
\mathscr{K}_{q}(g)=\sum_{k=0}^{\infty} \frac{\hat{g}(k)}{(\mu)_{k}} F^{k} q .
$$

By a computation, using that $F^{*}=-E$, or by Schur's lemma,

$$
\left\|\mathscr{K}_{q}(g)\right\|_{\nu}^{2}=\|g\|_{\mu}^{2}\|q\|_{\nu}^{2}
$$

Thus we may write (8) as

$$
\bigotimes_{i=1}^{n} \mathscr{A}^{\nu_{i}} \simeq \bigoplus_{s=0}^{\infty} \mathscr{A}^{|\nu|+2 s} \otimes Q_{s},
$$

where $Q_{s}$ is the space of highest weight polynomials of degree $s$ and $g \otimes q$ corresponds to $\mathscr{K}_{q}(g)$. (When all the $\nu_{i}$ are equal this is the decomposition with respect to $\tilde{G} \times S_{n}$, where $S_{n}$ is the symmetric group.) The space $\mathscr{H}_{s}$ of the previous section equals the space of finite sums of kernels $\mathscr{K}_{q}(g)$, where $q$ is a highest weight polynomial of degree $s$ and $g \in \mathscr{A}^{\mu}$.

Note that $\mathscr{K}_{q}(g)$ can be defined also for many analytic $g$ which are not in $\mathscr{A}^{\mu}$. We will denote by $H=H_{g}^{q}$ (for Hankel) the form on $\mathscr{A}^{\nu_{1}} \times \cdots \times \mathscr{A}^{\nu_{n}}$ with kernel $\mathscr{K}_{q}(g)$. This is a Hankel form of order $s$, and intertwining in the sense that $\left(H_{g}^{q}\right) * \phi=H_{g * \phi}^{q}$, where $H$ transforms as an element of $\bigotimes \mathscr{A}^{\nu}$ and $g$ as an element of $\mathscr{A}^{\mu}$. A general Hankel form of order $s$ can be written as a finite sum of forms $H_{g}^{q}$.

We denote by $\mathscr{J}_{q}: \mathscr{A}^{\nu_{1}} \times \cdots \times \mathscr{A}^{\nu_{n}} \rightarrow \mathscr{A}^{\mu}$ the $n$-linear operator defined by

$$
\mathscr{J}_{q}\left(f_{1}, \ldots, f_{n}\right)=\mathscr{K}_{q}^{*}\left(f_{1} \otimes \cdots \otimes f_{n}\right),
$$

where $\mathscr{K}_{q}^{*}: \bigotimes \mathscr{A}^{\nu} \rightarrow \mathscr{A}^{\mu}$ is the Hilbert space adjoint of $\mathscr{K}_{q}$. We call the operators $\mathscr{J}_{q}$ transvectants. In Proposition 3.2 we will see that $\mathscr{J}_{q}$ is a differential operator of the same degree as $q$. In the rest of this section we will write for short

$$
\begin{gathered}
K=\mathscr{K}_{q}(g), \\
J=\mathscr{J}_{q}\left(f_{1}, \ldots, f_{n}\right) .
\end{gathered}
$$

Thus we may write

$$
H_{g}^{q}\left(f_{1}, \ldots, f_{n}\right)=\left\langle f_{1} \otimes \cdots \otimes f_{n}, K\right\rangle_{\nu}=\langle J, g\rangle_{\mu} .
$$

To find explicit expressions for $J$ and $K$, we first observe that 


$$
J(w)=\left\langle J, k_{w}\right\rangle_{\mu}=\left\langle f_{1} \otimes \cdots \otimes f_{n}, \mathscr{K}_{q}\left(k_{w}\right)\right\rangle_{\nu} .
$$

But since

$$
k_{w}(z)=\frac{1}{(1-\bar{w} z)^{\mu}}=\sum_{k=0}^{\infty} \frac{(\mu)_{k} \bar{w}^{k}}{k !} z^{k},
$$

it follows from (10) that

$$
\mathscr{K}_{q}\left(k_{w}\right)=\sum_{k=0}^{\infty} \frac{\bar{w}^{k}}{k !} F^{k} q=e^{\bar{w} F} q,
$$

which gives

$$
J(w)=\left\langle f_{1} \otimes \cdots \otimes f_{n}, e^{\bar{w} F} q\right\rangle_{\nu} .
$$

Since $\left(e^{\bar{w} F}\right)^{*}=e^{-w E}$ we may also write

$$
J(w)=\left\langle e^{-w E}\left(f_{1} \otimes \cdots \otimes f_{n}\right), q\right\rangle_{\nu},
$$

provided that $e^{-w E}\left(f_{1} \otimes \cdots \otimes f_{n}\right) \in \otimes \mathscr{A}^{\nu}$. Now one may check that

$$
\begin{aligned}
& e^{\bar{w} F}\left(f_{1} \otimes \cdots \otimes f_{n}\right) \\
& =f_{1}\left(\frac{z_{1}}{1-\bar{w} z_{1}}\right) \cdots f_{n}\left(\frac{z_{n}}{1-\bar{w} z_{n}}\right) \frac{1}{\left(1-\bar{w} z_{1}\right)^{\nu_{1}} \cdots\left(1-\bar{w} z_{n}\right)^{\nu_{n}},} \\
& e^{-w E}\left(f_{1} \otimes \cdots \otimes f_{n}\right)=f_{1}\left(z_{1}+w\right) \cdots f_{n}\left(z_{n}+w\right) .
\end{aligned}
$$

(In fact, for any $X \in \mathfrak{s l}(2, \mathrm{C}), e^{w X}$ acts as in (5).) This gives integral formulas for $\mathscr{J}_{q}$ and $\mathscr{K}_{q}$. If some of the $\nu_{i}$ are less than 1 , the formulas of the following proposition are valid if properly reinterpreted (the integrals must be replaced by sums such as (2) and (4)).

Proposition 3.1. The operators $\mathscr{J}_{q}$ and $\mathscr{K}_{q}$ are given by

$$
\begin{aligned}
& J(w)=\int_{\mathbf{D}^{n}} q\left(\frac{z_{1}}{1-\bar{w} z_{1}}, \ldots, \frac{z_{n}}{1-\bar{w} z_{n}}\right) \frac{f_{1}\left(z_{1}\right) \cdots f_{n}\left(z_{n}\right)}{\left(1-w \overline{z_{1}}\right)^{\nu_{1}} \cdots\left(1-w \overline{z_{n}}\right)^{\nu_{n}}} d m_{\nu}(\mathbf{z}) \\
& K(\mathbf{z})=\int_{\mathbf{D}} q\left(\frac{z_{1}}{1-\bar{w} z_{1}}, \ldots, \frac{z_{n}}{1-\bar{w} z_{n}}\right) \frac{g(w)}{\left(1-\bar{w} z_{1}\right)^{\nu_{1}} \cdots\left(1-\bar{w} z_{n}\right)^{\nu_{n}}} d m_{\mu}(w),
\end{aligned}
$$

where $K$ and $J$ are as in (12) and (13). If $f_{1} \otimes \cdots \otimes f_{n}$ can be extended to the polydisc

$$
\mathbf{D}_{w}^{n}=\left\{\mathbf{z} \in \mathbf{C}^{n}|| z_{i}-w \mid<1,1 \leq i \leq n\right\},
$$

we may also write 


$$
\begin{aligned}
J(w) & =\int_{\mathbf{D}^{n}} f_{1}\left(z_{1}+w\right) \cdots f_{n}\left(z_{n}+w\right) \overline{q\left(z_{1}, \ldots, z_{n}\right)} d m_{\nu}(\mathbf{z}) \\
& =\int_{\mathbf{D}_{w}^{n}} f_{1}\left(z_{1}\right) \cdots f_{n}\left(z_{n}\right) \overline{q\left(z_{1}, \ldots, z_{n}\right)} d m_{\nu}\left(z_{1}-w, \ldots, z_{n}-w\right) .
\end{aligned}
$$

Proof. The formulas (15) and (17) follow from the corresponding formulas in the preceeding paragraph. It is also clear that (16) follows from (15), and (18) is immediate from (17) and (9).

It is not hard to check the intertwining properties of $\mathscr{J}_{q}$ and $\mathscr{K}_{q}$ directly from (15) and (16). Using the invariance of the scalar products and the identity

$$
1-\phi z_{i} \overline{\phi z_{j}}=\left(1-z_{i} \bar{z}_{j}\right) \phi^{\prime}\left(z_{i}\right)^{1 / 2}{\overline{\phi^{\prime}\left(z_{j}\right)}}^{1 / 2}, \quad \phi \in \tilde{G}
$$

one verifies that they reduce to the fact that

$$
q\left(\frac{z_{1}}{1-\bar{w} z_{1}}, \ldots, \frac{z_{n}}{1-\bar{w} z_{n}}\right)=q\left(\frac{\phi z_{1}}{1-\overline{\phi w} \phi z_{1}}, \ldots, \frac{\phi z_{n}}{1-\overline{\phi w} \phi z_{n}}\right){\overline{\phi^{\prime}(w)}}^{s} .
$$

This follows in turn from the properties of $q$ and the two identities

$$
\begin{gathered}
\phi z_{i}-\phi z_{j}=\left(z_{i}-z_{j}\right) \phi^{\prime}\left(z_{i}\right)^{1 / 2} \phi^{\prime}\left(z_{j}\right)^{1 / 2}, \\
\frac{z_{i}}{1-\bar{w} z_{i}}-\frac{z_{j}}{1-\bar{w} z_{j}}=\frac{z_{i}-z_{j}}{\left(1-\bar{w} z_{i}\right)\left(1-\bar{w} z_{j}\right)} .
\end{gathered}
$$

We will now generalize the expression (6) to the multilinear case.

Proposition 3.2. If $q(\mathbf{z})=\sum_{|\mathbf{s}|=s} c_{\mathbf{s}} \mathbf{z}^{\mathbf{s}}$ is a highest weight polynomial, then

$$
\mathscr{J}_{q}\left(f_{1}, \ldots, f_{n}\right)=\sum_{|\mathbf{s}|=s} \bar{c}_{\mathbf{s}} \frac{f_{1}^{\left(s_{1}\right)} \cdots f_{n}^{\left(s_{n}\right)}}{\left(\nu_{1}\right)_{s_{1}} \cdots\left(\nu_{n}\right)_{s_{n}}} .
$$

Proof. It suffices to take the $f_{i}$ as polynomials, so that (14) is valid. By Taylor's formula, $e^{-w E}\left(f_{1} \otimes \cdots \otimes f_{n}\right)$ is given by

$$
f_{1}\left(z_{1}+w\right) \cdots f_{n}\left(z_{n}+w\right)=\sum_{s_{1}, \ldots, s_{n} \geq 0} \frac{f_{1}^{\left(s_{1}\right)}(w) \cdots f_{n}^{\left(s_{n}\right)}(w)}{s_{1} ! \cdots s_{n} !} z_{1}^{s_{1}} \cdots z_{n}^{s_{n}} .
$$

Inserting this in (14) we obtain (19).

Alternatively, we may differentiate the reproducing formula (3) $k$ times to obtain

$$
\frac{f^{(k)}(w)}{(\nu)_{k}}=\int_{\mathbf{D}} \frac{\bar{z}^{k} f(z)}{(1-\bar{z} w)^{\nu+k}} d m_{\nu}(z)
$$


and use this to see that (19) is equivalent to (15). Note also that for $n=2$ we have up to a constant only $q\left(z_{1}, z_{2}\right)=\left(z_{1}-z_{2}\right)^{s}$, and then $\mathscr{J}_{q}\left(=\mathscr{J}_{s}\right)$ is given by (6).

Let us now return to the topic of integral formulas. It is sometimes convenient to express our generalized Hankel forms as boundary integrals. One has then only to apply the easily verified formula

$$
\langle f, g\rangle_{\mu}=\langle f, b\rangle_{1}=\frac{1}{2 \pi} \int_{\partial \mathbf{D}} f \bar{b}|d w|,
$$

where

$$
b(z)=\sum_{k=0}^{\infty} \frac{k !}{(\mu)_{k}} \hat{g}(k) z^{k}
$$

It follows that

$$
H_{g}^{q}\left(f_{1}, \ldots, f_{n}\right)=\frac{1}{2 \pi} \int_{\partial \mathbf{D}} J \bar{b}|d w|
$$

and that

$$
K(\mathbf{z})=\frac{1}{2 \pi} \int_{\partial \mathbf{D}} q\left(\frac{z_{1}}{1-\bar{w} z_{1}}, \ldots, \frac{z_{n}}{1-\bar{w} z_{n}}\right) \frac{b(w)}{\left(1-\bar{w} z_{1}\right)^{\nu_{1}} \cdots\left(1-\bar{w} z_{n}\right)^{\nu_{n}}}|d w| .
$$

This is of particular interest when $\mu$ is an integer. We can then write

$$
g(z)=\frac{h^{(\mu-1)}(z)}{(\mu-1) !}
$$

where $h(z)=z^{\mu-1} b(z)$. The function $h$ is interesting because it also behaves nicely under the group action, a fact that has been called Bol's lemma (cf. [22] for a historical discussion):

$$
\left(\frac{d}{d z}\right)^{\mu-1}\left(h(\phi z) \phi^{\prime}(z)^{(2-\mu) / 2}\right)=h^{(\mu-1)}(\phi z) \phi^{\prime}(z)^{\mu / 2} .
$$

This reflects the fact that $\mathscr{A}^{\mu}$ is equivalent as a representation to a space of analytic functions modulo polynomials of degree $<\mu-1$, transforming according to

$$
U\left(\phi^{-1}\right) h=h(\phi z) \phi^{\prime}(z)^{(2-\mu) / 2},
$$

and an isomorphism is given by $h^{(\mu-1)} \mapsto h$. Our kernel can be expressed as

$$
K(\mathbf{z})=\frac{1}{2 \pi i} \int_{\partial \mathbf{D}} q\left(\frac{z_{1}}{1-\bar{w} z_{1}}, \ldots, \frac{z_{n}}{1-\bar{w} z_{n}}\right) \frac{h(w) \bar{w}^{\mu}}{\left(1-\bar{w} z_{1}\right)^{\nu_{1}} \cdots\left(1-\bar{w} z_{n}\right)^{\nu_{n}}} d w
$$


In [12], higher order Hankel forms were introduced through Newton's divided differences

$$
h\left(z_{1}, z_{2}\right)=\frac{h\left(z_{1}\right)-h\left(z_{2}\right)}{z_{1}-z_{2}}, h\left(z_{1}, z_{2}, z_{3}\right)=\frac{h\left(z_{1}, z_{2}\right)-h\left(z_{1}, z_{3}\right)}{z_{2}-z_{3}} \text { etc. }
$$

Let us see how to find expressions involving these. We now assume that all the $\nu_{i}$ (and hence $\mu$ ) are positive integers. First suppose that $q$ is of the form

$$
q(\mathbf{z})=\prod_{i, j}\left(z_{i}-z_{j}\right)^{s_{i j}} .
$$

We let $s_{i}=\sum_{j}\left(s_{i j}+s_{j i}\right)$, so that $\sum_{i} s_{i}=2 s$. In that case we find that

$$
\begin{aligned}
& K(\mathbf{z})=\frac{1}{2 \pi i} \int_{\partial \mathbf{D}} \prod_{i, j}\left(\frac{z_{i}-z_{j}}{\left(1-\bar{w} z_{i}\right)\left(1-\bar{w} z_{j}\right)}\right)^{s_{i j}} \frac{h(w) \bar{w}^{\mu}}{\left(1-\bar{w} z_{1}\right)^{\nu_{1}} \cdots\left(1-\bar{w} z_{n}\right)^{\nu_{n}}} d w \\
& =\frac{q(\mathbf{z})}{2 \pi i} \int_{\partial \mathbf{D}} \frac{h(w)}{\left(w-z_{1}\right)^{s_{1}+\nu_{1}} \cdots\left(w-z_{n}\right)^{S_{n}+\nu_{n}}} d w \\
& =q(\mathbf{z}) h(\overbrace{\underbrace{z_{1}, \ldots, z_{1}}_{s_{1}+\nu_{1}}, \ldots, \underbrace{z_{n}, \ldots, z_{n}}_{s_{n}+\nu_{n}}}^{\mu}),
\end{aligned}
$$

using a well-known integral formula for the divided differences. If $q$ is not of the form (22), we get a linear combination of such expressions.

To summarize, a Hankel form $H_{g}^{q}$ of order $s$ is given by

$$
H_{g}^{q}\left(f_{1}, \ldots, f_{n}\right)=\left\langle f_{1} \otimes \cdots \otimes f_{n}, K\right\rangle_{\nu}=\langle J, g\rangle_{\mu}=\langle J, b\rangle_{1},
$$

where $J$ is given by (19), $K=K_{g}$ by (16) and $K=K_{b}$ by (20). For integer $\mu$ we can write $K=K_{h}$ as in (21), and when all the $\nu_{i}$ are integers it is given by a linear combination of expressions like (23).

\section{Trace class criterion.}

We will now discuss $\mathfrak{\Xi}_{p}$-properties of our forms. In the case $\sum \nu_{i}-\max \nu_{i}>1$ and $p \geq 1$ we obtain a complete trace class criterion. Our proof generalizes the one for the case $n=0, s=0, \nu_{1}=\nu_{2}>1$ (that is for little Hankel operators on Dzhrbashyan spaces) given in [18]. For the theory of trace classes of multilinear forms we refer to [7], though we will denote by $\Xi_{\infty}$ the space of bounded forms, not the compact ones. Note that $\Xi_{2}$ is just the space $\otimes \mathscr{A}^{\nu}$ (when viewed as a space of forms). For $s \in \mathrm{R}$ and $0<p \leq \infty$, we introduce the Besov space $\mathscr{B}^{s, p}$ of analytic functions on the disc such that 


$$
\left(1-|z|^{2}\right)^{m-s}\left|f^{(m)}(z)\right| \in L^{p}\left(\frac{d x d y}{1-|z|^{2}}\right),
$$

where $m$ is any natural number with $m>s$. Then $\mathscr{A}^{\nu}=\mathscr{B}^{\frac{1}{2}(1-\nu), 2}$. This definition is independent of $m$, that is

$$
f \in \mathscr{B}^{s, p} \Leftrightarrow f^{\prime} \in \mathscr{B}^{s-1, p} \quad\left(\text { so } f \in \mathscr{A}^{\nu} \Leftrightarrow f^{\prime} \in \mathscr{A}^{\nu+2}\right) .
$$

Also, $\mathscr{B}^{\frac{1}{p}-\frac{\nu}{2}, p}$ is invariant under the action (1) for any $\nu \in \mathrm{R}$. We finally let $\mathscr{B}_{0}^{s}$ be the closure of the polynomials in $\mathscr{B}^{s, \infty}$; then (with $m$ as above)

$$
f \in \mathscr{B}_{0}^{s} \Leftrightarrow \lim _{|z| \nearrow 1}\left(1-|z|^{2}\right)^{m-s}\left|f^{(m)}(z)\right|=0 .
$$

The main step in the proof of our trace class criterion is Lemma 4.1 below. It is also true in the case $\nu=0$, when it is due to Arazy and Fisher [3]. It was observed in [18] that it can be extended to other values of $\nu$. We will deduce the lemma from the atomic decomposition of analytic Besov spaces as given by Peloso in [24], generalizing earlier work by Coifman and Rochberg [8].

Lemma 4.1. Let $X$ be a Banach space of analytic functions on $\mathbf{D}$ such that $1 \in X$ and the norm of $X$ is invariant under the action (1) for some $\nu>0$. Then $\mathscr{B}^{1-\frac{\nu}{2}, 1}$ is continuously contained in $X$.

Proof. By [24], for any $\beta>0$ there exists a sequence $\left(w_{i}\right)_{i=1}^{\infty}$ in D and a constant $C$ such that any function $f \in \mathscr{B}^{1-\frac{\nu}{2}, 1}$ can be decomposed as

$$
f(z)=\sum_{i=1}^{\infty} c_{i} \frac{\left(1-\left|w_{i}\right|^{2}\right)^{\beta}}{\left(1-z \bar{w}_{i}\right)^{\beta+\frac{\nu}{2}}}, \quad \text { where } \quad \sum_{i=1}^{\infty}\left|c_{i}\right| \leq C\|f\|_{\mathscr{B}^{1-\frac{\nu}{2}, 1}} .
$$

We take $\beta=\nu / 2$, so that this can be written as $f=\sum c_{i}\left(1 * \phi_{i}\right)$, with $\phi_{i} z=\left(z-w_{i}\right) /\left(1-\bar{w}_{i} z\right)$. Then

$$
\|f\|_{X} \leq \sum_{i=1}^{\infty}\left|c_{i}\right|\left\|1 * \phi_{i}\right\|_{X} \leq C\|1\|_{X}\|f\|_{\mathscr{B}^{1-\frac{\nu}{2}, 1}}
$$

which proves the lemma.

Lemma 4.2. For $\nu_{i}>0(1 \leq i \leq n)$ and $\sum \nu_{i}-\max \nu_{i}>1$ we have

$$
f_{i} \in \mathscr{A}^{\nu_{i}} \Rightarrow f_{1} \cdots f_{n} \in \mathscr{B}^{1-\frac{|\nu|}{2}, 1} .
$$

Proof. First let us show that

$$
\mathscr{A}^{\nu} \subseteq \mathscr{B}^{\frac{1}{-}-\frac{\nu}{2}, p} \quad(2 \leq p \leq \infty, \nu>0) .
$$

(This is a version of Sobolev's imbedding theorem.) Since 


$$
f \in \mathscr{B}^{\frac{1}{-}-\frac{\nu}{2}, p} \Leftrightarrow\left(1-|z|^{2}\right)^{\frac{1}{2}(\nu+2)}\left|f^{\prime}(z)\right| \in L^{p}\left(\frac{d x d y}{\left(1-|z|^{2}\right)^{2}}\right)
$$

it suffices to prove this when $p=\infty$. But in that case the inclusion follows from

$$
|f(z)|=\left|\left\langle f, k_{z}\right\rangle_{\nu}\right| \leq\|f\|_{\nu}\left(k_{z}(z)\right)^{\frac{1}{2}}=\|f\|_{\nu}\left(1-|z|^{2}\right)^{-\frac{\nu}{2}} .
$$

Using (25) one sees that it suffices to prove the lemma when $\max \nu_{i}=\nu_{1}>2$, and thus $\nu_{2}+\cdots+\nu_{n}>1$. We can then choose $p_{2}, \ldots, p_{n}$ with $1 / p_{2}+\cdots+1 / p_{n}=1 / 2$ and $p_{i}>2 / \nu_{i}(2 \leq i \leq n)$, which gives, by Hölder's inequality,

$$
\begin{aligned}
& \int_{\mathbf{D}}\left|f_{1} \cdots f_{n}\right|\left(1-|z|^{2}\right)^{\frac{1}{2}\left(\nu_{1}+\cdots+\nu_{n}\right)^{-2}} d x d y \\
& \leq\left(\int_{\mathbf{D}}\left|f_{1}\right|^{2}\left(1-|z|^{2}\right)^{\nu_{1}-2} d x d y\right)^{\frac{1}{2}} \prod_{i=2}^{n}\left(\int_{\mathbf{D}}\left|f_{i}\right|_{i}^{p}\left(1-|z|^{2}\right)^{\frac{1}{2} \nu_{i} p_{i}-2} d x d y\right)^{\frac{1}{p_{i}}} .
\end{aligned}
$$

This completes the proof since $f_{i} \in \mathscr{B}^{1 / p_{i}-\nu_{i} / 2, p_{i}}$, and the parameters are chosen so that one may take $m=0$ in (24).

We can now state

THeORem 4.3. Let $H$ be a Hankel form of order $s$ in $\mathscr{A}^{\nu_{1}} \otimes \cdots \otimes \mathscr{A}^{\nu_{n}}$, written as in the previous section

$$
H=\sum_{j=1}^{k} H_{g_{j}}^{q_{j}}
$$

where $k=\left(\begin{array}{c}n+s-2 \\ n-2\end{array}\right)$ and $\left(q_{1}, \ldots, q_{k}\right)$ is any basis for the space of highest weight polynomials of degree $s$, and let $\mu=|\nu|+2 s$. If $\sum \nu_{i}-\max \nu_{i}>1$, we have the equivalences

$$
\begin{gathered}
H \in \mathfrak{\Xi}_{p} \Leftrightarrow g_{j} \in \mathfrak{B}^{\frac{1}{-}-\frac{\mu}{2}, p} \text { for all } j(1 \leq p \leq \infty), \\
H \text { is compact } \Leftrightarrow g_{j} \in \mathscr{B}_{0}^{-\frac{\mu}{2}} \text { for all } j .
\end{gathered}
$$

If $\nu_{i}>0$ are arbitrary, we have in (26) the implication $\Leftarrow$ for $1 \leq p \leq 2$ and $\Rightarrow$ for $2 \leq p \leq \infty$ and in (27) the implication $\Rightarrow$.

Proof. We fix $j$, put $q=q_{j}$ and let $X=\left\{g \mid H_{g}^{q} \in \Xi_{1}\right\}$. This is a Banach space with norm $\|g\|_{X}=\left\|H_{g}^{q}\right\|_{\mathfrak{S}_{1}}$, which is invariant when $g$ transforms as an element of $\mathscr{A}^{\mu}$. Since $g=1$ gives the form with kernel $q$ which is trivially in $\Im_{1}$, Lemma 4.1 gives $\mathscr{B}^{1-\frac{\mu}{2}, 1} \subseteq X$. This shows that 


$$
g_{j} \in \mathscr{B}^{1-\frac{\mu}{2}, 1} \text { for all } j \Rightarrow H \in \Xi_{1} .
$$

Assume conversely that $H \in \mathfrak{S}_{1}$, and that $\sum \nu_{i}-\max \nu_{i}>1$. We want to show that

$$
H \in \mathfrak{\Xi}_{1} \Rightarrow g_{j} \in \mathscr{B}^{1-\frac{\mu}{2}, 1}
$$

for a fixed $j$. We may then assume that $\left(q_{1}, \ldots, q_{k}\right)$ is orthogonal. By definition, one can write

$$
H\left(f_{1}, \ldots, f_{n}\right)=\sum_{i=1}^{\infty} c_{i}\left\langle f_{1}, \psi_{1}^{i}\right\rangle_{\nu_{1}} \cdots\left\langle f_{n}, \psi_{n}^{i}\right\rangle_{\nu_{n}},
$$

where the $\psi_{j}^{i}$ satisfy

$$
\sum_{i=1}^{\infty}\left|c_{i}\right|\left\|\psi_{1}^{i}\right\|_{\nu_{1}} \cdots\left\|\psi_{n}^{i}\right\|_{\nu_{n}}<\infty
$$

Equivalently,

$$
\sum_{i=1}^{k} \mathscr{K}_{q_{i}}\left(g_{i}\right)=\sum_{i=1}^{\infty} c_{i}\left(\psi_{1}^{i} \otimes \cdots \otimes \psi_{n}^{i}\right) .
$$

We now apply the equality

$$
\mathscr{J}_{q_{j}} \mathscr{K}_{q_{i}}=\left\langle q_{i}, q_{j}\right\rangle_{\nu} \mathrm{Id}
$$

which is just the polarization of (11). It gives

$$
g_{j}=\|q\|_{\nu}^{-2} \sum_{i=1}^{\infty} c_{i} \mathscr{J}_{q}\left(\psi_{1}^{i}, \ldots, \psi_{n}^{i}\right)
$$

(where still $q=q_{j}$ ), so it suffices to prove that, for some constant $C$,

$$
\left\|\mathscr{J}_{q}\left(\psi_{1}^{i}, \ldots, \psi_{n}^{i}\right)\right\|_{\mathscr{B}^{1-\frac{\mu}{2}, 1}} \leq C\left\|\psi_{1}^{i}\right\|_{\nu_{1}} \cdots\left\|\psi_{n}^{i}\right\|_{\nu_{n}} .
$$

Now if $\left(s_{1}, \ldots, s_{n}\right)$ is a multi-index of length $s$, we clearly have

$$
\sum\left(\nu_{i}+2 s_{i}\right)-\max \left(\nu_{i}+2 s_{i}\right)>1 \text {. }
$$

But then (29) follows from Lemma 4.2 and (25), so the theorem holds for $p=1$.

Now, since $\mathfrak{S}_{1}^{*}=\mathfrak{\Xi}_{\infty}$ and $\left(\mathscr{B}^{1-\frac{\mu}{2}, 1}\right)^{*}=\mathscr{B}^{-\frac{\mu}{2}, \infty}$ with respect to the dualities given by $\Xi_{2}$ and $\mathscr{A}^{\mu}$ respectively, the theorem follows for $p=\infty$ from the case $p=1$, using (28), and then for general $p$ by interpolation. The com- 
pactness criterion follows since the space of compact forms is the closure in $\mathfrak{\Im}_{\infty}$ of the forms with polynomial kernels.

What happens when the $\nu_{i}$ are so small that there is no equivalence in Theorem 4.3? This seems to be much more difficult. One certainly has to go beyond the scale of Besov spaces, since for $n=2, s=0$ and $\nu_{1}=\nu_{2}=1, H$ is bounded if and only if $g \in \mathrm{BMO}$. We refer to [32] for trace ideal criteria for Hankel type operators in this situation.

\section{Orthogonal polynomials.}

So far we have formulated our theory only for the unit disc D. However, it applies to any Riemann surface $U$ conformally equivalent to $\mathbf{D}$. One has only to define

$$
\mathscr{A}^{\nu}(U)=\left\{(f \circ \psi)\left(\psi^{\prime}\right)^{\nu / 2} \mid f \in \mathscr{A}^{\nu}(\mathbf{D})\right\} \quad(\nu>0),
$$

where $\psi: U \rightarrow \mathbf{D}$ is a conformal isomorphism. This space is independent of $\psi$. We carry over the Hilbert space structure and get a unitary representation as before. When $U$ is a disc, a half-plane or a strip the objects in study can be expressed using Fourier transform on the boundary. In the case $\mathscr{A}^{\nu} \otimes \mathscr{A}^{\nu}$ $(\nu \geq 1)$, this was worked out in [19]. The orthogonality of the decomposition then leads naturally to three systems of orthogonal polynomials. The rest of this paper will be concerned with generalizations of these systems to the multilinear case.

Let us write down expressions for the norm in $\mathscr{A}^{\nu}$ of the half-plane $\{\operatorname{Im} z>0\}$ and the strip $\{0<\operatorname{Im} z<\pi\}$ respectively:

$$
\begin{aligned}
& \|f\|_{\nu}^{2}=\frac{\Gamma(\nu)}{2 \pi} \int_{0}^{\infty}|\hat{f}(\xi)|^{2} \xi^{1-\nu} \frac{d \xi}{2 \pi}, \\
& \|f\|_{\nu}^{2}=\Gamma(\nu) \int_{-\infty}^{\infty}|\hat{f}(\xi)|^{2} \frac{e^{-\pi \xi}}{\left|\Gamma\left(\frac{\nu}{2}+i \xi\right)\right|^{2}} \frac{d \xi}{2 \pi} .
\end{aligned}
$$

Here the hat denotes Fourier transform of (in general distributional) boundary values on the real axis. This can be found in [19], except that for the strip one there finds the alternative expression

$$
\|f\|_{\nu}^{2}=\frac{(\nu-1) 2^{\nu-2}}{\pi} \int_{-\infty}^{\infty}|\hat{f}(\xi)|^{2} \int_{0}^{\pi} e^{-2 y \xi}(\sin y)^{\nu-2} d y \frac{d \xi}{2 \pi} \quad(\nu>1) .
$$

The inner integral can, however, be expressed as indicated (cf. [31], formula 3.4.6(5)), which also gives an extension to all positive $\nu$. Since 
$\hat{f}_{y}(\xi)=e^{-y \xi} \hat{f}(\xi)$, where $f_{y}(x)=f(x+i y)$, (30) can also be written more symmetrically as

$$
\|f\|_{\nu}^{2}=\Gamma(\nu) \int_{0}^{\infty}\left(\left|\hat{f}_{0}(\xi)\right|^{2}+\left|\hat{f}_{\pi}(-\xi)\right|^{2}\right) \frac{e^{-\pi \xi}}{\left|\Gamma\left(\frac{\nu}{2}+i \xi\right)\right|^{2}} \frac{d \xi}{2 \pi} .
$$

The expressions for higher order Hankel forms given in [19] extend immediately to the multilinear case, so we will just write them down. It is convenient to use the symbol corresponding to $b$. In the halfplane, disc and strip respectively, they are then given by (using the same somewhat arbitrary normalization as in [19])

$$
H\left(f_{1}, \ldots, f_{n}\right)=\int_{\mathrm{R}_{+}^{n}} \overline{\hat{b}\left(\xi_{1}+\cdots+\xi_{n}\right) p(\xi)} \hat{f}_{1}\left(\xi_{1}\right) \cdots \hat{f}_{n}\left(\xi_{n}\right) \frac{d \xi}{(2 \pi)^{n}},
$$

$$
\begin{aligned}
& \text { (32) } H\left(f_{1}, \ldots, f_{n}\right)=\sum_{k=0}^{\infty} \overline{\hat{b}(k)} \sum_{m_{1}+\cdots+m_{n}=k+s} \overline{\mathfrak{p}\left(m_{1}, \ldots, m_{n}\right)} \hat{f}_{1}\left(m_{1}\right) \cdots \hat{f}_{n}\left(m_{n}\right), \\
& H\left(f_{1}, \ldots, f_{n}\right)=\int_{\mathrm{R}^{n}} \frac{\hat{b}\left(\xi_{1}+\cdots+\xi_{n}\right) \mathbf{p}(\xi)\left(1+e^{-2 \pi\left(\xi_{1}+\cdots+\xi_{n}\right)}\right)}{f_{1}\left(\xi_{1}\right) \cdots \hat{f}_{n}\left(\xi_{n}\right) \frac{d \xi}{(2 \pi)^{n}},}
\end{aligned}
$$

where

$$
p=T q, \quad \mathfrak{p}=\mathfrak{T} q, \quad \mathbf{p}=\mathbf{T} q
$$

and $T, \mathfrak{I}$ and $\mathbf{T}$ are the operators sending $z_{1}^{s_{1}} \cdots z_{n}^{s_{n}}$ to

$$
\begin{aligned}
& \frac{\xi_{1}^{s_{1}} \cdots \xi_{n}^{s_{n}}}{\left(\nu_{1}\right)_{s_{1}} \cdots\left(\nu_{n}\right)_{s_{n}}}, \quad(-1)^{s} \frac{\left(-m_{1}\right)_{s_{1}} \cdots\left(-m_{n}\right)_{s_{n}}}{\left(\nu_{1}\right)_{s_{1}} \cdots\left(\nu_{n}\right)_{s_{n}}}, \\
& (-1)^{s} \frac{\left(\frac{\nu_{1}}{2}-i \xi_{1}\right)_{s_{1}} \cdots\left(\frac{\nu_{n}}{2}-i \xi_{n}\right)_{s_{n}}}{\left(\nu_{1}\right)_{s_{1}} \cdots\left(\nu_{n}\right)_{s_{n}}}
\end{aligned}
$$

respectively. Note that $p, \mathfrak{p}$ and $\mathbf{p}$ are polynomials of degree $s$. Now if $q$ and $q^{\prime}$ are two orthogonal highest weight polynomials, then the corresponding Hankel forms are orthogonal for all $g$. It follows that the polynomials $p=T q, \ldots, \mathbf{p}^{\prime}=\mathbf{T} q^{\prime}$ satisfy the orthogonality relations

$$
\int p(\xi) \overline{p^{\prime}(\xi)} \xi_{1}^{\nu_{1}-1} \cdots \xi_{n}^{\nu_{n}-1} d \xi=0
$$

where the integration is over $\xi_{1}+\cdots+\xi_{n}=C, \xi_{1}, \ldots, \xi_{n}>0$ and $C>0$ is arbitrary, 


$$
\sum_{m_{1}+\cdots+m_{n}=M} \mathfrak{p}\left(m_{1}, \ldots, m_{n}\right) \overline{\mathfrak{p}^{\prime}\left(m_{1}, \ldots, m_{n}\right)} \frac{\left(\nu_{1}\right)_{m_{1}} \cdots\left(\nu_{n}\right)_{m_{n}}}{m_{1} ! \cdots m_{n} !}=0
$$

for $M=s, s+1, \ldots$ and

$$
\int \mathbf{p}(\xi) \overline{\mathbf{p}^{\prime}(\xi)}\left|\Gamma\left(\frac{\nu_{1}}{2}+i \xi_{1}\right)\right|^{2} \cdots\left|\Gamma\left(\frac{\nu_{n}}{2}+i \xi_{n}\right)\right|^{2} d \xi=0,
$$

where the integration is over the entire plane $\xi_{1}+\cdots+\xi_{n}=C$ and $C \in \mathrm{R}$ is arbitrary.

When $q$ is of the form (22) we have found an amusing Rodrigues-type formula for $p=T q$ :

$$
p(\xi)=\frac{(-1)^{s} \xi_{1}^{1-\nu_{1}} \cdots \xi_{n}^{1-\nu_{n}}}{\left(\nu_{1}\right)_{s_{1}} \cdots\left(\nu_{n}\right)_{s_{n}}} q\left(\frac{\partial}{\partial \xi}\right)\left(\xi_{1}^{\nu_{1}+s_{1}-1} \cdots \xi_{n}^{\nu_{n}+s_{n}-1}\right)
$$

(where $\left.s_{i}=\sum_{j}\left(s_{i j}+s_{j i}\right)\right)$. To see this, write

$$
q(\mathbf{z})=\sum_{|\mathbf{t}|=t} c_{\mathbf{t}} \mathbf{z}^{\mathbf{t}}, \quad p(\xi)=\sum_{|\mathbf{t}|=t} c_{\mathbf{t}} \frac{\xi_{1}^{t_{1}} \cdots \xi_{n}^{t_{n}}}{\left(\nu_{1}\right)_{t_{1}} \cdots\left(\nu_{n}\right)_{t_{n}}} .
$$

Then (35) reduces to $c_{\mathrm{t}}=(-1)^{s} c_{\mathrm{s}-\mathrm{t}}$, that is to the immediately verified identity

$$
z_{1}^{s_{1}} \cdots z_{n}^{s_{n}} q\left(\frac{1}{z_{1}}, \ldots, \frac{1}{z_{n}}\right)=(-1)^{s} q\left(z_{1}, \ldots, z_{n}\right) .
$$

We remark that in the case when $p$ and $p^{\prime}$ have different homogeneity, (33) follows directly from (35) by partial integration.

The polynomials $p, \mathfrak{p}$ and $\mathbf{p}$ have a "group-theoretical" meaning. In fact they are (generalized) Clebsch-Gordan coefficients of our representation. To understand this, first note that Fourier expansion in our three cases means expansion in eigenfunctions of certain one-parameter subgroups of $G$, which are given by affine automorphisms. For the unit disc we have the rotations, that is the elliptic subgroup of $G$, for the half-plane and the strip the translations which form the parabolic and hyperbolic subgroup respectively. (The hyperbolic subgroup can also be represented as dilations of the half-plane; one then has the Fourier transform on $\left(R_{+}, \times\right)$, that is the Mellin transform.) Let us consider the case $n=2$. We know two orthogonal bases of $\mathscr{A}^{\nu_{1}} \otimes \mathscr{A}^{\nu_{2}}$, namely $\left(z_{1}^{i} z_{2}^{j}\right)_{i, j=0}^{\infty}$, that is the tensor products of eigenfunctions of the elliptic subgroup, and $\left(F^{k}\left(z_{1}-z_{2}\right)^{l}\right)_{k, l=0}^{\infty}$. The change between these is given by 


$$
\begin{aligned}
F^{k}\left(z_{1}-z_{2}\right)^{l} & =\sum_{i+j=k+l} \frac{\left(\nu_{1}\right)_{i}\left(\nu_{2}\right)_{j}}{i ! j !}\left\langle z_{1}^{i} z_{2}^{j}, F^{k}\left(z_{1}-z_{2}\right)^{l}\right\rangle_{\nu} z_{1}^{i} z_{2}^{j} \\
& =k ! \sum_{i+j=k+l} \frac{\left(\nu_{1}\right)_{i}\left(\nu_{2}\right)_{j}}{i ! j !} H\left(z_{1}^{i}, z_{2}^{j}\right) z_{1}^{i} z_{2}^{j},
\end{aligned}
$$

where $H$ is the Hankel form of order $l$ given by $q=\left(z_{1}-z_{2}\right)^{l}$ and $b=w^{k}$. Comparing with (32) we find that

$$
F^{k}\left(z_{1}-z_{2}\right)^{l}=k ! \sum_{i+j=k+l} \frac{\left(\nu_{1}\right)_{i}\left(\nu_{2}\right)_{j}}{i ! j !}(\mathfrak{I} q)(i, j) z_{1}^{i} z_{2}^{j},
$$

so the polynomial $\mathfrak{p}=\mathfrak{I} q$ gives the matrix for a change between our two bases. Similarly the polynomials $T q$ and $\mathrm{T} q$ appear in the integral kernel for the (continuous) expansion of $F^{k}\left(z_{1}-z_{2}\right)^{l}$ in tensor products of eigenfunctions of the parabolic and hyperbolic subgroups respectively. This means that, when $n=2$, our three classes of polynomials agree after normalization with the Clebsch-Gordan coefficients, with respect to the three subgroups, of our representation. These can (when the $\nu_{i}$ are integers) be found in [31], where it is shown that they are given by Hahn polynomials, Jacobi polynomials and "Hahn polynomials of imaginary argument" respectively. So, in particular, the polynomials of [19] must agree with those.

In the case $n=2$, two highest weight polynomials are orthogonal precisely if they have different degree of homogeneity, so there is essentially one system of orthogonal polynomials in each of the three cases. In the case $n \geq 3$ we may get different systems starting with different bases in the space of highest weight polynomials. In Section 6 we will consider the non-orthogonal basis

$$
q_{\mathbf{s}}(\mathbf{z})=\left(z_{1}-z_{n}\right)^{s_{1}} \cdots\left(z_{n-1}-z_{n}\right)^{s_{n-1}}, \quad \mathbf{s}=\left(s_{1}, \ldots, s_{n-1}\right) .
$$

In that case the polynomials $T q_{\mathrm{s}}, \mathfrak{I} q_{\mathrm{s}}$ and $\mathbf{T} q_{\mathrm{s}}$ can be expressed in terms of a single (generalized) hypergeometric function. It turns out that for $n=3$ the polynomials $T q_{\mathrm{s}}$ where introduced by Appell in [1], see also the book [2]. So we call the polynomials that appear generalized Appell polynomials. In Section 7 we will consider the case when one starts with an orthogonal basis of the space of highest weight polynomials, so that application of $T, \mathfrak{I}$ and $\mathbf{T}$ gives complete systems of orthogonal polynomials. Expressions for these in terms of hypergeometric functions are more complicated.

\section{Generalized Appell polynomials.}

In this section we will study $T q_{\mathrm{s}}, \mathfrak{I} q_{\mathrm{s}}$ and $\mathbf{T} q_{\mathrm{s}}$ for $q_{\mathrm{s}}$ given by (36), where $\mathbf{s}$ is a multi-index of length $s$. Note that since $T$, $\mathfrak{I}$ and $\mathbf{T}$ commute with permu- 
tations of the variables, our results extend to the bases $\prod_{j \neq i}\left(z_{j}-z_{i}\right)^{s_{j}}$ $(1 \leq i \leq n-1)$. Let us start with the case of the half-plane. The polynomials $p_{\mathrm{s}}=T q_{\mathrm{s}}$ can then be expressed in terms of the Lauricella function $F_{A}^{(n-1)}$, for which we recall the definition

$$
F_{A}^{(n)}\left(\begin{array}{c}
a, b_{1}, \ldots, b_{n} \\
c_{1}, \ldots, c_{n}
\end{array} \mid x_{1}, \ldots, x_{n}\right)=\sum_{k_{1}, \ldots, k_{n}=0}^{\infty} \frac{(a)_{|k|}\left(b_{1}\right)_{k_{1}} \cdots\left(b_{n}\right)_{k_{n}}}{k_{1} ! \cdots k_{n} !\left(c_{1}\right)_{k_{1}} \cdots\left(c_{n}\right)_{k_{n}}} x_{1}^{k_{1}} \cdots x_{n}^{k_{n}},
$$

where $|k|=k_{1}+\cdots+k_{n}$ (cf. [29] or [31]). Indeed, from the expansion

$$
q_{\mathbf{s}}(\mathbf{z})=\sum_{k_{1}, \ldots, k_{n-1}=0}^{\infty} \frac{\left(-s_{1}\right)_{k_{1}} \cdots\left(-s_{n-1}\right)_{k_{n-1}}}{k_{1} ! \cdots k_{n-1} !}(-1)^{s} z_{1}^{k_{1}} \cdots z_{n-1}^{k_{n-1}} z_{n}^{s-|k|}
$$

we obtain, writing $\left(\nu_{n}\right)_{s-|k|}=(-1)^{|k|}\left(\nu_{n}\right)_{s} /\left(1-\nu_{n}-s\right)_{|k|}$, that

$$
\begin{aligned}
& p_{\mathbf{s}}(\xi)=\sum_{k_{1}, \ldots, k_{n}-1=0}^{\infty} \frac{\left(-s_{1}\right)_{k_{1}} \cdots\left(-s_{n-1}\right)_{k_{n-1}}(-1)^{s} \xi_{1}^{k_{1}} \cdots \xi_{n-1}^{k_{n}-1} \xi_{n}^{s-|k|}}{k_{1} ! \cdots k_{n-1} !\left(\nu_{1}\right)_{k_{1}} \cdots\left(\nu_{n-1}\right)_{k_{n-1}}\left(\nu_{n}\right)_{s-|k|}} \\
& =\frac{\left(-\xi_{n}\right)^{s}}{\left(\nu_{n}\right)_{s}} \sum_{k_{1, \ldots, k_{n}-1=0}}^{\infty} \frac{\left(1-\nu_{n}-s\right)_{|k|}\left(-s_{1}\right)_{k_{1}} \cdots\left(-s_{n-1}\right)_{k_{n-1}}}{k_{1} ! \cdots k_{n-1} !\left(\nu_{1}\right)_{k_{1}} \cdots\left(\nu_{n-1}\right)_{k_{n-1}}}\left(-\frac{\xi_{1}}{\xi_{n}}\right)^{k_{1}} \cdots\left(-\frac{\xi_{n-1}}{\xi_{n}}\right)^{k_{n-1}} \\
& =\frac{\left(-\xi_{n}\right)^{s}}{\left(\nu_{n}\right)_{s}} F_{A}^{(n-1)}\left(\begin{array}{c}
1-\nu_{n}-s,-s_{1}, \ldots,-s_{n-1} \\
\nu_{1}, \ldots, \nu_{n-1}
\end{array} \mid-\frac{\xi_{1}}{\xi_{n}}, \ldots,-\frac{\xi_{n-1}}{\xi_{n}}\right) .
\end{aligned}
$$

If we fix $C$ and assume that $\xi_{1}+\cdots+\xi_{n}=C$, we may eliminate $\xi_{n}$ and get orthogonal polynomials in $n-1$ variables. Without loss of generality we take $C=1$. The polynomials

$$
\tilde{p}_{\mathbf{s}}\left(\xi_{1}, \ldots, \xi_{n-1}\right)=p_{\mathbf{s}}\left(\xi_{1}, \ldots, \xi_{n-1}, 1-\xi_{1}-\cdots-\xi_{n-1}\right)
$$

are then, for different values of $|\mathbf{s}|=s$, orthogonal on

$$
\xi_{1}+\cdots+\xi_{n-1} \leq 1, \quad \xi_{1}, \ldots, \xi_{n-1}>0
$$

with respect to the weight

$$
\xi_{1}^{\nu_{1}-1} \cdots \xi_{n-1}^{\nu_{n-1}-1}\left(1-\xi_{1}-\cdots-\xi_{n-1}\right)^{\nu_{n}-1} .
$$

One easily obtains a Rodrigues formula for these polynomials:

$$
\begin{aligned}
& \tilde{p}_{\mathbf{s}}\left(\xi_{1}, \ldots, \xi_{n}-1\right)=\frac{(-1)^{s} \xi_{1}^{1-\nu_{1}} \cdots \xi_{n-1}^{1-\nu_{n-1}}\left(1-\xi_{1}-\cdots-\xi_{n-1}\right)^{1-\nu_{n}}}{\left(\nu_{1}\right)_{s_{1}} \cdots\left(\nu_{n-1}\right)_{s_{n-1}}\left(\nu_{n}\right)_{s}} \\
& \times \frac{\partial^{s}}{\partial \xi_{1}^{S_{1}} \cdots \partial \xi_{n-1}^{s_{n-1}}}\left(\xi_{1}^{\nu_{1}+s_{1}-1} \cdots \xi_{n-1}^{\nu_{n-1}+s_{n-1}-1}\left(1-\xi_{1}-\cdots-\xi_{n-1}\right)^{\nu_{n}+s-1}\right) .
\end{aligned}
$$


For $n=3$ they are as remarked above studied in [2]. In the notation of that book we have in this case

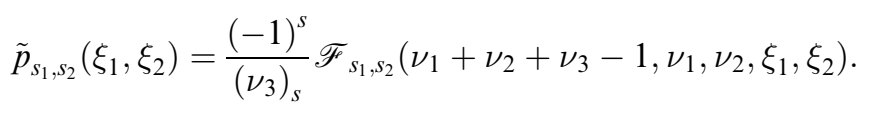

For $n=2$ we recognize the Jacobi polynomials

$$
\tilde{p}_{s}(\xi)=\frac{(-1)^{s}}{\left(\nu_{2}\right)_{s}} \mathscr{F}_{s}\left(\nu_{1}+\nu_{2}-1, \nu_{1}, \xi\right)=\frac{(-1)^{s} s !}{\left(\nu_{1}\right)_{s}\left(\nu_{2}\right)_{s}} P_{s}^{\left(\nu_{1}-1, \nu_{2}-1\right)}(1-2 \xi),
$$

in the notation of [2] and of most modern authors [17, 31] respectively. When $\nu_{1}=\nu_{2}$ we get Gegenbauer polynomials as in [19].

We now turn to the corresponding polynomials for the disc, that is $\mathfrak{p}_{\mathrm{s}}=\mathfrak{I} q_{\mathrm{s}}$, with $q_{\mathrm{s}}$ as before. For different values of $s=|\mathbf{s}|$, these polynomials are orthogonal in the sense of (34). They can be expressed in terms of one of Karlsson's generalized Kampé de Fériet functions [29], namely

$$
\begin{gathered}
F_{1: 1}^{1: 2}\left(\begin{array}{c}
a: b_{1}, c_{1} ; \ldots ; b_{n}, c_{n} \\
d: e_{1} ; \ldots ; e_{n}
\end{array} \mid x_{1}, \ldots, x_{n}\right) \\
=\sum_{k_{1}, \ldots, k_{n}=0}^{\infty} \frac{(a)_{|k|}\left(b_{1}\right)_{k_{1}} \cdots\left(b_{n}\right)_{k_{n}}\left(c_{1}\right)_{k_{1}} \cdots\left(c_{n}\right)_{k_{n}}}{k_{1} ! \cdots k_{n} !(d)_{|k|}\left(e_{1}\right)_{k_{1}} \cdots\left(e_{n}\right)_{k_{n}}} x_{1}^{k_{1}} \cdots x_{n}^{k_{n}}
\end{gathered}
$$

(with $n$ replaced by $n-1$ ). In fact, the same calculation as above gives

$$
\begin{aligned}
& \mathfrak{p}_{\mathbf{s}}\left(m_{1}, \ldots, m_{n}\right) \\
& =\frac{\left(-m_{n}\right)_{s}}{\left(\nu_{n}\right)_{s}} \sum_{k_{1}, \ldots, k_{n}-1=0}^{\infty} \frac{\left(-s_{1}\right)_{k_{1}} \cdots\left(-s_{n-1}\right)_{k_{n-1}}\left(-m_{1}\right)_{k_{1}} \cdots\left(-m_{n-1}\right)_{k_{n-1}}\left(1-\nu_{n}-s\right)_{|k|}}{k_{1} ! \cdots k_{n-1} !\left(\nu_{1}\right)_{k_{1}} \cdots\left(\nu_{n-1}\right)_{k_{n-1}}\left(1+m_{n}-s\right)_{|k|}} \\
& =\frac{\left(-m_{n}\right)_{s}}{\left(\nu_{n}\right)_{s}} F_{1: 1}^{1: 2}\left(\begin{array}{c}
\left.1-\nu_{n}-s:-s_{1},-m_{1} ; \ldots ;-s_{n-1},-m_{n-1} \mid 1, \ldots, 1\right) . \\
1+m_{n}-s: \nu_{1} ; \ldots ; \nu_{n-1}
\end{array} \mid\right.
\end{aligned}
$$

When $n=2$ we get the function ${ }_{3} F_{2}$,

$$
\begin{aligned}
\mathfrak{p}_{s}\left(m_{1}, m_{2}\right) & =\frac{\left(-m_{2}\right)_{s}}{\left(\nu_{2}\right)_{s}} \sum_{k=0}^{\infty} \frac{(-s)_{k}\left(-m_{1}\right)_{k}\left(1-\nu_{2}-s\right)_{k}}{k !\left(\nu_{1}\right)_{k}\left(1+m_{2}-s\right)_{k}} \\
& =\frac{\left(-m_{2}\right)_{s}}{\left(\nu_{2}\right)_{s}}{ }_{3} F_{2}\left(\begin{array}{c}
-s,-m_{1}, 1-\nu_{2}-s \\
\nu_{1}, 1+m_{2}-s
\end{array} \mid 1\right) .
\end{aligned}
$$

Applying the transformation formula

$$
{ }_{3} F_{2}\left(\begin{array}{c}
-s, a, b \\
c, d
\end{array} \mid 1\right)=\frac{(b)_{s}(d-a)_{s}}{(c)_{s}(d)_{s}}{ }_{3} F_{2}\left(\begin{array}{c}
-s, c-b, 1-d-s \\
1-b-s, 1+a-d-s
\end{array} \mid 1\right)
$$


and the expression (2.7.19) in [17] for the Hahn polynomials

$$
h_{n}^{(\alpha, \beta)}(x, N)=\frac{(-1)^{n}}{n !}(N-n)_{n}(\beta+1)_{n}{ }_{3} F_{2}\left(\begin{array}{c}
-n, \alpha+\beta+n+1,-x \\
\beta+1,1-N
\end{array} \mid 1\right)
$$

we find that

$$
\mathfrak{p}_{s}\left(m_{1}, m_{2}\right)=\frac{(-1)^{s} s !}{\left(\nu_{1}\right)_{s}\left(\nu_{2}\right)_{s}} h_{s}^{\left(\nu_{1}-1, \nu_{2}-1\right)}\left(m_{2}, m_{1}+m_{2}+1\right) .
$$

Finally, in the case of the strip we have in the same way

$$
\begin{aligned}
& \mathfrak{p}_{\mathbf{s}}\left(\xi_{1}, \ldots, \xi_{n}\right)=\mathbf{T} q_{\mathbf{s}}\left(\xi_{1}, \ldots, \xi_{n}\right)=\frac{\left(\frac{1}{2} \nu_{n}-i \xi_{n}\right)_{s}}{\left(\nu_{n}\right)_{s}} \\
& \times F_{1: 1}^{1: 2}\left(\begin{array}{c}
1-\nu_{n}-s:-s_{1}, \frac{1}{2} \nu_{1}-i \xi_{1} ; \ldots ;-s_{n-1}, \frac{1}{2} \nu_{n-1}-i \xi_{n-1} \mid 1, \ldots, 1 \\
1-\frac{1}{2} \nu_{n}-s+i \xi_{n}: \nu_{1} ; \ldots ; \nu_{n-1}
\end{array} \mid .\right.
\end{aligned}
$$

For $n=2$ this reads

$$
\mathfrak{p}_{s}\left(\xi_{1}, \xi_{2}\right)=\frac{\left(\frac{1}{2} \nu_{2}-i \xi_{2}\right)_{s}}{\left(\nu_{2}\right)_{s}}{ }_{3} F_{2}\left(\begin{array}{c|c}
-s, \frac{1}{2} \nu_{1}-i \xi_{1}, 1-\nu_{2}-s \\
\nu_{1}, 1-\frac{1}{2} \nu_{2}-s+i \xi_{2}
\end{array} \mid 1\right)
$$

which is, in agreement with [31], a Hahn polynomial of imaginary argument

\section{Complete orthogonal systems.}

If one replaces the $q_{\mathrm{s}}$ of the previous section by an orthogonal basis of the space of highest weight polynomials, it will give an orthogonal decomposition of the space $\mathscr{H}_{s}$ into $\left(\begin{array}{c}n+s-2 \\ n-2\end{array}\right)$ parts. Applying the operators $T, \mathfrak{I}$ and $\mathbf{T}$ to such a basis one obtains three orthogonal systems of polynomials. This can be done in many ways, and there is no "canonical" one. There is however a method that allows us to construct many different bases, and we then have the problem how to change between these. Related questions have received much attention from phycisists (cf. [5]), especially for the group $\mathrm{SO}(3$ ) (or its double cover $\mathrm{SU}(2)$ ).

To construct our bases we use a "binary coupling" technique. If $q_{1}$ is a polynomial of highest weight $-\mu_{1}$ in $\mathscr{A}^{\nu_{1}} \otimes \cdots \otimes \mathscr{A}^{\nu_{m}}$ and $q_{2}$ one of highest weight $-\mu_{2}$ in $\mathscr{A}^{\nu_{m+1}} \otimes \cdots \otimes \mathscr{A}^{\nu_{n}}$ (where $1 \leq m \leq n-1$ ), then we define

$$
\begin{aligned}
\left(q_{1}, q_{2}\right)_{s} & =\left(\mu_{1}\right)_{s}\left(\mu_{2}\right)_{s}\left(\mathscr{K}_{q_{1}} \otimes \mathscr{K}_{q_{2}}\right)\left(z_{1}-z_{2}\right)^{s} \\
& =\sum_{k=0}^{s}\left(\begin{array}{l}
s \\
k
\end{array}\right)(-1)^{s-k} \frac{\left(\mu_{1}\right)_{s}\left(\mu_{2}\right)_{s}}{\left(\mu_{1}\right)_{k}\left(\mu_{2}\right)_{s-k}} F^{k} q_{1} \otimes F^{s-k} q_{2} .
\end{aligned}
$$

Here $\mathscr{K}_{q_{1}}: \mathscr{A}^{\mu_{1}} \rightarrow \bigotimes_{i=1}^{m} \mathscr{A}^{\nu_{i}}$ and similarly for $\mathscr{K}_{q_{2}}$, and the factor $\left(\mu_{1}\right)_{s}\left(\mu_{2}\right)_{s}$ 
is introduced for convenience. From the properties of $\mathscr{K}$ follows that $\left(q_{1}, q_{2}\right)_{s}$ is a polynomial of highest weight $-\left(2 s+\mu_{1}+\mu_{2}\right)$ in $\mathscr{A}^{\nu_{1}} \otimes \cdots \otimes \mathscr{A}^{\nu_{n}}$. (This is the well-known Clebsch-Gordan formula.) Equivalently, $\left(q_{1}, q_{2}\right)_{s}$ is the unique highest weight polynomial such that

$$
\mathscr{J}_{\left(q_{1}, q_{2}\right)_{s}}\left(f_{1}, \ldots, f_{n}\right)=\left(\mu_{1}\right)_{s}\left(\mu_{2}\right)_{s} \mathscr{J}_{s}\left(\mathscr{J}_{q_{1}}\left(f_{1}, \ldots, f_{m}\right), \mathscr{J}_{q_{2}}\left(f_{m+1}, \ldots, f_{n}\right)\right) \text {, }
$$

where $\mathscr{J}_{s}=\mathscr{J}_{\left(z_{1}-z_{2}\right)^{s}}$ is given by (6), with $\nu_{i}$ replaced by $\mu_{i}(i=1,2)$.

An integral formula (with the obvious interpretation in the case when $\mu_{1}$ or $\mu_{2}$ is small) follows from (16):

$$
\begin{aligned}
& \left(q_{1}, q_{2}\right)_{s}(\mathbf{z}) \\
& =\int_{\mathbf{D} \times \mathbf{D}} q_{1}\left(\frac{z_{1}}{1-\bar{w}_{1} z_{1}}, \ldots, \frac{z_{m}}{1-\bar{w}_{1} z_{m}}\right) q_{2}\left(\frac{z_{m+1}}{1-\bar{w}_{2} z_{m+1}}, \ldots, \frac{z_{n}}{1-\bar{w}_{2} z_{n}}\right) \\
& \times \frac{\left(\mu_{1}\right)_{s}\left(\mu_{2}\right)_{s}\left(w_{1}-w_{2}\right)^{s} d m_{\mu_{1}}\left(w_{1}\right) d m_{\mu_{2}}\left(w_{2}\right)}{\left(1-\bar{w}_{1} z_{1}\right)^{\nu_{1}} \cdots\left(1-\bar{w}_{1} z_{m}\right)^{\nu_{m}}\left(1-\bar{w}_{2} z_{m+1}\right)^{\nu_{m}+1} \cdots\left(1-\bar{w}_{2} z_{n}\right)^{\nu_{n}}} .
\end{aligned}
$$

Writing, for example,

$$
\begin{aligned}
& \mathscr{A}^{\nu_{1}} \otimes \mathscr{A}^{\nu_{2}} \otimes \mathscr{A}^{\nu_{3}}=\left(\mathscr{A}^{\nu_{1}} \otimes \mathscr{A}^{\nu_{2}}\right) \otimes \mathscr{A}^{\nu_{3}} \\
= & \bigoplus_{s=0}^{\infty} \mathscr{A}^{\nu_{1}+\nu_{2}+2 s} \otimes \mathscr{A}^{\nu_{3}}=\bigoplus_{s, t=0}^{\infty} \mathscr{A}^{\nu_{1}+\nu_{2}+\nu_{3}+2 s+2 t},
\end{aligned}
$$

we see that the polynomials $\left(\left(1_{\nu_{1}}, 1_{\nu_{2}}\right)_{s}, 1_{\nu_{3}}\right)_{t}$ for $s, t=0,1,2, \ldots$, where $1_{\nu_{i}}=1 \in \mathscr{A}^{\nu_{i}}$, form an orthogonal basis of the space of highest weight polynomials, and thus

$$
\left(F^{k}\left(\left(1_{\nu_{1}}, 1_{\nu_{2}}\right)_{s}, 1_{\nu_{3}}\right)_{t}\right)_{k, s, t=0}^{\infty}
$$

an orthogonal basis of $\mathscr{A}^{\nu_{1}} \otimes \mathscr{A}^{\nu_{2}} \otimes \mathscr{A}^{\nu_{3}}$. To describe the bases constructible in this way, it will be convenient to use labeled binary trees (as is done in similar contexts e.g. in $[5,17,31])$. Instead of giving formal definitions, we will explain the concepts needed by means of examples.

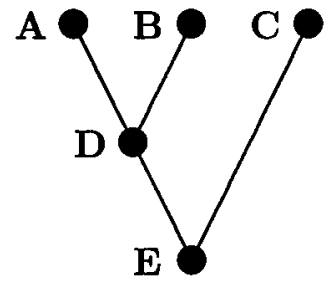

Figure 1a

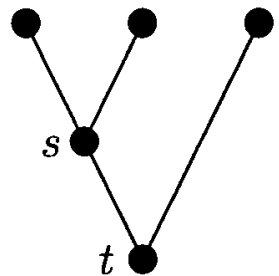

Figure $1 b$

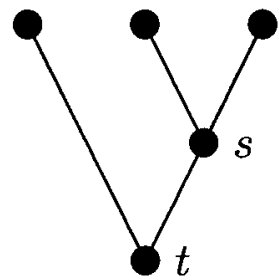

Figure 1c 


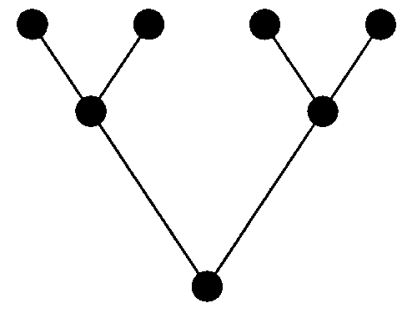

Figure 2a

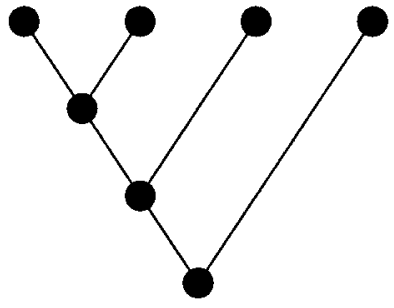

Figure 2b

Figure 1a pictures an unlabeled binary tree with three terminal points $\mathbf{A}, \mathbf{B}$ and $\mathbf{C}$ and two bifurcation points $\mathbf{D}$ and $\mathbf{E}$. A tree with $n$ terminal points has $n-1$ bifurcation points. In a labeled binary tree a natural number is attached to each bifurcation point. To each labeled tree we associate a highest weight polynomial. Trees $1 \mathrm{~b}$ and $1 \mathrm{c}$ then correspond to

$$
\left(\left(1_{\nu_{1}}, 1_{\nu_{2}}\right)_{s}, 1_{\nu_{3}}\right)_{t} \text { and }\left(1_{\nu_{1}},\left(1_{\nu_{2}}, 1_{\nu_{3}}\right)_{s}\right)_{t}
$$

respectively, where $s$ and $t$ are natural numbers. Each unlabeled tree with $n$ terminal points gives the orthogonal basis of the highest weight polynomials in $\mathscr{A}^{\nu_{1}} \otimes \cdots \otimes \mathscr{A}^{\nu_{n}}$ (or of the space itself, by applying $F^{k}$ ) corresponding to all possible labellings of the tree, so for example trees $2 \mathrm{a}$ and $2 \mathrm{~b}$ give the bases

$F^{k}\left(\left(1_{\nu_{1}}, 1_{\nu_{2}}\right)_{s},\left(1_{\nu_{3}}, 1_{\nu_{4}}\right)_{t}\right)_{u}$ and $F^{k}\left(\left(\left(1_{\nu_{1}}, 1_{\nu_{2}}\right)_{s}, 1_{\nu_{3}}\right)_{t}, 1_{\nu_{4}}\right)_{u} \quad(k, s, t, u=0,1, \ldots)$ of $\bigotimes_{i=1}^{4} \mathscr{A}^{\nu_{i}}$ respectively. One can construct other bases by permuting the variables. For example we can first form the polynomial $\left(\left(1_{\nu_{1}}, 1_{\nu_{3}}\right)_{s}, 1_{\nu_{2}}\right)_{t}$ in $\mathscr{A}^{\nu_{1}} \otimes \mathscr{A}^{\nu_{3}} \otimes \mathscr{A}^{\nu_{2}}$ and then permute the last two variables to get back to $\mathscr{A}^{\nu_{1}} \otimes \mathscr{A}^{\nu_{2}} \otimes \mathscr{A}^{\nu_{3}}$. Our results below extend immediately to such polynomials, though for simplicity we state them only for the case when the spaces $\mathscr{A}^{\nu_{i}}$ are taken in order.

It is easy to compute the norm of one of these polynomials, so that we can normalize our bases. From (39) and (11) follows that

$$
\begin{aligned}
\left\|\left(q_{1}, q_{2}\right)_{s}\right\|^{2} & =\left(\mu_{1}\right)_{s}^{2}\left(\mu_{2}\right)_{s}^{2}\left\|q_{1}\right\|^{2}\left\|q_{2}\right\|^{2}\left\|\left(z_{1}-z_{2}\right)^{s}\right\|_{\mathscr{A}^{\mu_{1}} \otimes \mathscr{A}^{\mu_{2}}} \\
& =\left(\mu_{1}\right)_{s}^{2}\left(\mu_{2}\right)_{s}^{2}\left\|q_{1}\right\|^{2}\left\|q_{2}\right\|^{2} \sum_{k=0}^{s}\left(\begin{array}{l}
s \\
k
\end{array}\right)^{2} \frac{k !(s-k) !}{\left(\mu_{1}\right)_{k}\left(\mu_{2}\right)_{s-k}} .
\end{aligned}
$$

The sum can be computed using the Chu-Vandermonde formula (see below), which gives

$$
\left\|\left(q_{1}, q_{2}\right)_{s}\right\|^{2}=s !\left(\mu_{1}\right)_{s}\left(\mu_{2}\right)_{s}\left(\mu_{1}+\mu_{2}+s-1\right)_{s}\left\|q_{1}\right\|^{2}\left\|q_{2}\right\|^{2} .
$$


If $q$ is given by a tree, one thus obtains a product formula for $\|q\|$, with one factor for each bifurcation point.

We will now consider the problem of giving explicit expressions for our polynomials as functions of $z_{i}-z_{j}$. There are of course infinitely many ways of doing this, but we have found one particularly symmetric and transparent expression, which is given as Theorem 7.2 below. Let us begin with a special case in the form of a lemma.

Lemma 7.1. The polynomial $q=\left(q_{1}, q_{2}\right)_{s}$, where $q_{1}=1 \in \mathscr{A}^{\nu_{1}} \otimes \cdots \otimes \mathscr{A}^{\nu_{m}}$, $q_{2}=1 \in \mathscr{A}^{\nu_{m+1}} \otimes \cdots \otimes \mathscr{A}^{\nu_{n}}(1 \leq m \leq n-1)$ can be expressed as

$$
q(\mathbf{z})=\sum_{k_{i j}} s !\left(\nu_{1}\right)_{k_{1}} \cdots\left(\nu_{n}\right)_{k_{n}} \prod_{1 \leq i \leq m, m+1 \leq j \leq n} \frac{\left(z_{i}-z_{j}\right)^{k_{i j}}}{k_{i j} !},
$$

where the sum is over all natural numbers $k_{i j}$ with $1 \leq i \leq m, m+1 \leq j \leq n$ such that $\sum_{i=1}^{m} \sum_{j=m+1}^{n} k_{i j}=s$, and where

$$
k_{i}= \begin{cases}\sum_{j=m+1}^{n} k_{i j} & 1 \leq i \leq m, \\ \sum_{j=1}^{m} k_{j i} & m+1 \leq i \leq n .\end{cases}
$$

Proof. By (41) we have

$$
q(\mathbf{z})=\int_{\mathbf{D}^{2}} \frac{\left(\mu_{1}\right)_{s}\left(\mu_{2}\right)_{s}\left(w_{1}-w_{2}\right)^{s} d m_{\mu_{1}}\left(w_{1}\right) d m_{\mu_{2}}\left(w_{2}\right)}{\left(1-\bar{w}_{1} z_{1}\right)^{\nu_{1}} \cdots\left(1-\bar{w}_{1} z_{m}\right)^{\nu_{m}}\left(1-\bar{w}_{2} z_{m+1}\right)^{\nu_{m+1}} \cdots\left(1-\bar{w}_{2} z_{n}\right)^{\nu_{n}}},
$$

where $\mu_{1}=\nu_{1}+\cdots+\nu_{m}, \quad \mu_{2}=\nu_{m+1}+\cdots+\nu_{n}$. Using the expansion $(1-z)^{-a}=\sum_{k=0}^{\infty}(a)_{k} z^{k} / k !$ in the denominator we get the expression

$$
q(\mathbf{z})=\sum_{p_{1}+\cdots+p_{n}=s} \frac{s !\left(\mu_{1}\right)_{s}\left(\mu_{2}\right)_{s}\left(\nu_{1}\right)_{p_{1}} \cdots\left(\nu_{n}\right)_{p_{n}}(-1)^{p_{m+1}+\cdots+p_{n}}}{p_{1} ! \cdots p_{n} !\left(\mu_{1}\right)_{p_{1}+\cdots+p_{m}}\left(\mu_{2}\right)_{p_{m+1}+\cdots+p_{n}}} z_{1}^{p_{1}} \cdots z_{n}^{p_{n}} .
$$

The lemma follows from this by applying the binomial theorem to each of the factors $\left(z_{i}-z_{j}\right)^{k_{i j}}$ in (43).

For the sake of clarity, let us illustrate this by considering the case $m=2, n=4$. We then write (43) as 


$$
\begin{aligned}
& \sum_{k_{13}+k_{23}+k_{14}+k_{24}=s} \frac{s !}{k_{13} ! k_{23} ! k_{14} ! k_{24} !}\left(\nu_{1}\right)_{k_{13}+k_{14}}\left(\nu_{2}\right)_{k_{23}+k_{24}}\left(\nu_{3}\right)_{k_{13}+k_{23}}\left(\nu_{4}\right)_{k_{14}+k_{24}} \\
\times & \left(z_{1}-z_{3}\right)^{k_{13}}\left(z_{1}-z_{4}\right)^{k_{14}}\left(z_{2}-z_{3}\right)^{k_{23}}\left(z_{2}-z_{4}\right)^{k_{24}} \\
= & \sum_{\substack{p_{13}+p_{23}+p_{14}+p_{24} \\
+p_{31}+p_{32}+p_{41}+p_{42}=s}} \frac{s !\left(\nu_{1}\right)_{p_{13}+p_{31}+p_{14}+p_{41}} \cdots\left(\nu_{4}\right)_{p_{14}+p_{41}+p_{24}+p_{42}}}{p_{13} ! p_{23} ! p_{14} ! p_{24} ! p_{31} ! p_{32} ! p_{41} ! p_{42} !} \\
\times & z_{1}^{p_{13}+p_{14}} z_{2}^{p_{23}+p_{24}}\left(-z_{3}\right)^{p_{31}+p_{32}}\left(-z_{4}\right)^{p_{41}+p_{42}} \\
= & \sum_{p_{1}+p_{2}+p_{3}+p_{4}=s} s !\left(\nu_{1}\right)_{p_{1}}\left(\nu_{2}\right)_{p_{2}}\left(\nu_{3}\right)_{p_{3}}\left(\nu_{4}\right)_{p_{4}}(-1)^{p_{3}+p_{4}} z_{1}^{p_{1}} z_{2}^{p_{2}} z_{3}^{p_{3}} z_{4}^{p_{4}} \\
\times & \sum_{\substack{p_{13}+p_{14}=p_{1}, p_{23}+p_{24}=p_{2}}} \frac{\left(\nu_{3}+p_{3}\right)_{p_{13}+p_{23}}\left(\nu_{4}+p_{4}\right)_{p_{14}+p_{24}} \sum_{p_{13} ! p_{23} ! p_{14} ! p_{24} !} \frac{\left(\nu_{1}+p_{1}\right)_{p_{31}+p_{41}}\left(\nu_{2}+p_{2}\right)_{p_{32}+p_{42}}}{p_{31}+p_{32}=p_{3},}}{p_{31} ! p_{32} ! p_{41} ! p_{42} !} .
\end{aligned}
$$

The equality then follows if we can prove that

$$
\sum_{\substack{p_{13}+p_{14}=p_{1} \\ p_{23}+p_{24}=p_{2}}} \frac{\left(\gamma_{1}\right)_{p_{13}+p_{23}}\left(\gamma_{2}\right)_{p_{14}+p_{24}}}{p_{13} ! p_{23} ! p_{14} ! p_{24} !}=\frac{\left(\gamma_{1}+\gamma_{2}\right)_{p_{1}+p_{2}}}{p_{1} ! p_{2} !}
$$

For general $m$ and $k=n-m$ one similarly arrives at (changing $p_{i, m+j}$ to $q_{i j}$ )

$$
\sum_{\substack{q_{11}+\cdots+q_{1 k}=p_{1}, q_{m 1}+\cdots+q_{m k}=p_{m}}} \frac{\left(\gamma_{1}\right)_{q_{11}+\cdots+q_{m 1}} \cdots\left(\gamma_{n}\right)_{q_{1 k}+\cdots+q_{m k}}}{q_{11} ! \cdots q_{m k} !}=\frac{\left(\gamma_{1}+\cdots+\gamma_{k}\right)_{p_{1}+\cdots+p_{m}}}{p_{1} ! \cdots p_{m} !} .
$$

(For $m=1, k=2$ this is the Chu-Vandermonde formula.) To prove it in general, expand both sides of

$$
\begin{aligned}
& \left(1-x_{1}-\cdots-x_{m}\right)^{-\left(\gamma_{1}+\cdots+\gamma_{k}\right)} \\
& =\left(1-x_{1}-\cdots-x_{m}\right)^{-\gamma_{1}} \cdots\left(1-x_{1}-\cdots-x_{m}\right)^{-\gamma_{k}}
\end{aligned}
$$

and identify the coefficient of $x_{1}^{p_{1}} \cdots x_{m}^{p_{m}}$.

To formulate our theorem, we need the concept of the bifurcation point where two terminal points of a binary tree meet. As before, we only indicate what we mean by an example: $\mathbf{A}$ and $\mathbf{C}$ in Figure 1 a meet at $\mathbf{E}$.

THEOREM 7.2. Let $q$ be a highest weight polynomial in $\mathscr{A}^{\nu_{1}} \otimes \cdots \otimes \mathscr{A}^{\nu_{n}}$ given by a binary tree with bifurcation points labeled $s_{1}, \ldots, s_{n-1}$, and with the terminal points numbered from left to right by $1, \ldots, n$. Then 


$$
q(\mathbf{z})=\sum_{k_{i j}} s_{1} ! \cdots s_{n-1} !\left(\nu_{1}\right)_{k_{1}} \cdots\left(\nu_{n}\right)_{k_{n}} \prod_{1 \leq i<j \leq n} \frac{\left(z_{i}-z_{j}\right)^{k_{i j}}}{k_{i j} !}
$$

where the sum is over all natural numbers $k_{i j}$ with $1 \leq i<j \leq n$ such that, for each bifurcation point $N$, the sum of all $k_{i j}$ such that $i$ and $j$ meet at $N$ equals the label of $N$, and where $k_{i}=\sum_{1 \leq j<i} k_{j i}+\sum_{i<j \leq n} k_{i j}(1 \leq i \leq n)$.

As an example, the polynomial $q=\left(\left(1_{\nu_{1}}, 1_{\nu_{2}}\right)_{s},\left(1_{\nu_{3}}, 1_{\nu_{4}}\right)_{t}\right)_{u}$ can be written as

$$
\begin{aligned}
q(\mathbf{z}) & =\left(z_{1}-z_{2}\right)^{s}\left(z_{3}-z_{4}\right)^{t} \sum_{\substack{k_{13}+k_{14} \\
+k_{23}+k_{24}=u}} \frac{u !}{k_{13} ! k_{14} ! k_{23} ! k_{24} !}\left(\nu_{1}\right)_{s+k_{13}+k_{14}}\left(\nu_{2}\right)_{s+k_{23}+k_{24}} \\
& \times\left(\nu_{3}\right)_{t+k_{13}+k_{23}}\left(\nu_{4}\right)_{t+k_{14}+k_{24}}\left(z_{1}-z_{3}\right)^{k_{13}}\left(z_{1}-z_{4}\right)^{k_{14}}\left(z_{2}-z_{3}\right)^{k_{23}}\left(z_{2}-z_{4}\right)^{k_{24}} .
\end{aligned}
$$

We now prove the theorem.

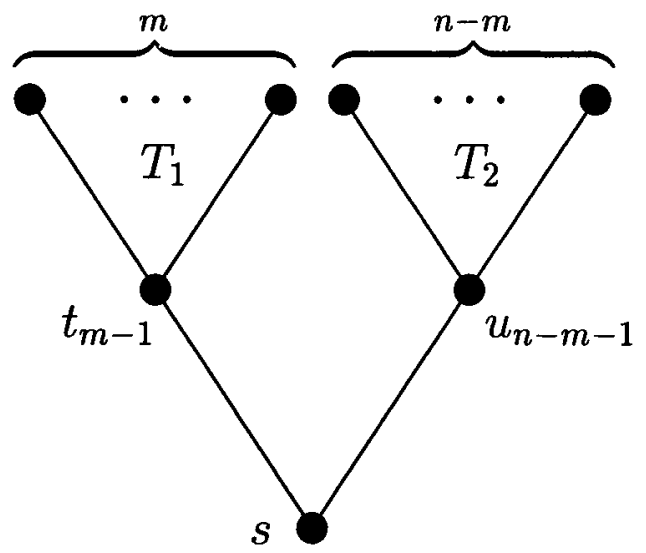

Figure 3

Proof. We will use induction on the tree. Suppose that $q=\left(q_{1}, q_{2}\right)_{s}$ is given by the tree in Figure 3, and that the theorem is true for the polynomials $q_{1}$ and $q_{2}$ given by the trees $T_{1}$ (labelled by $t_{1}, \ldots, t_{m-1}$ ) and $T_{2}$ (labelled by $\left.u_{1}, \ldots, u_{n-m-1}\right)$ respectively. We then have

$$
\begin{gathered}
q_{1}(\mathbf{z})=\sum_{k_{i j} \in A} t_{1} ! \cdots t_{m-1} !\left(\nu_{1}\right)_{k_{1}^{\prime}} \cdots\left(\nu_{m}\right)_{k_{m}^{\prime}} \prod_{1 \leq i<j \leq m} \frac{\left(z_{i}-z_{j}\right)^{k_{i j}}}{k_{i j} !}, \\
q_{2}(\mathbf{z})=\sum_{k_{i j} \in B} u_{1} ! \cdots u_{n-m-1} !\left(\nu_{m+1}\right)_{k_{m+1}^{\prime}} \cdots\left(\nu_{n}\right)_{k_{n}^{\prime}} \prod_{m+1 \leq i<j \leq n} \frac{\left(z_{i}-z_{j}\right)^{k_{i j}}}{k_{i j} !},
\end{gathered}
$$

where 


$$
k_{i}^{\prime}= \begin{cases}\sum_{1 \leq j<i} k_{j i}+\sum_{i<j \leq m} k_{i j} & 1 \leq i \leq m, \\ \sum_{m+1 \leq j<i} k_{j i}+\sum_{i<j \leq n} k_{i j} & m+1 \leq i \leq n\end{cases}
$$

and the sets $A$ and $B$ are determined by $T_{1}$ and $T_{2}$ according to the theorem. By (41),

$$
\begin{gathered}
q(\mathbf{z})=\sum_{k_{i j} \in A \cup B} t_{1} ! \cdots t_{m-1} ! u_{1} ! \cdots u_{n-m-1} !\left(\nu_{1}\right)_{k_{1}^{\prime}} \cdots\left(\nu_{n}\right)_{k_{n}^{\prime}} \prod_{\substack{1 \leq i<j \leq m \text { or } \\
m+1 \leq i<j \leq n}} \frac{\left(z_{i}-z_{j}\right)^{k_{i j}}}{k_{i j} !} \\
\times \int_{\mathbf{D}^{2}} \frac{\left(\mu_{1}\right)_{s}\left(\mu_{2}\right)_{s}\left(w_{1}-w_{2}\right)^{s} d m_{\mu_{1}}\left(w_{1}\right) d m_{\mu_{2}}\left(w_{2}\right)}{\left(1-\bar{w}_{1} z_{1}\right)^{\nu_{1}+k_{1}^{\prime}} \cdots\left(1-\bar{w}_{1} z_{m}\right)^{\nu_{m}+k_{m}^{\prime}}\left(1-\bar{w}_{2} z_{m+1}\right)^{\nu_{m+1}+k_{m+1}^{\prime} \cdots\left(1-\bar{w}_{2} z_{n}\right)^{\nu_{n}+k_{n}^{\prime}}},}
\end{gathered}
$$

where $\mu_{1}=\sum_{i=1}^{m} \nu_{i}+2 \sum_{i=1}^{m-1} t_{i}=\sum_{i=1}^{m} \nu_{i}+\sum_{i=1}^{m} k_{i}^{\prime}$ and analogously for $\mu_{2}$. By (41) again and Lemma 7.1, the integral is given by

$$
\sum_{\sum_{i=1}^{m} \sum_{j=m+1}^{n}} s !\left(\nu_{1}+k_{1}^{\prime}\right)_{k_{1}^{\prime \prime}} \cdots\left(\nu_{n}+k_{n}^{\prime}\right)_{k_{n}^{\prime \prime}} \prod_{1 \leq i \leq m, m+1 \leq j \leq n} \frac{\left(z_{i}-z_{j}\right)^{k_{i j}}}{k_{i j} !},
$$

where

$$
k_{i}^{\prime \prime}= \begin{cases}\sum_{j=m+1}^{n} k_{i j} & 1 \leq i \leq m, \\ \sum_{j=1}^{m} k_{j i} & m+1 \leq i \leq n .\end{cases}
$$

Writing $\left(\nu_{i}\right)_{k_{i}^{\prime}}\left(\nu_{i}+k_{i}^{\prime}\right)_{k_{i}^{\prime \prime}}=\left(\nu_{i}\right)_{k_{i}}$, one arrives at

$q(\mathbf{z})=\sum_{k_{i j} \in A \cup B} \sum_{\sum_{i=1}^{m} \sum_{j=m+1}^{n}} s ! t_{1} ! \cdots u_{n-m-1} !\left(\nu_{1}\right)_{k_{1}} \cdots\left(\nu_{n}\right)_{k_{n}} \prod_{1 \leq i<j \leq n} \frac{\left(z_{i}-z_{j}\right)^{k_{i j}}}{k_{i j} !}$,

where $k_{i}=k_{i}^{\prime}+k_{i}^{\prime \prime}$ is now as in the formulation of the theorem. Checking how $A$ and $B$ are defined one sees that this completes the proof.

We will now study the polynomials $T q$, $\mathfrak{I} q$ and $\mathbf{T} q$ where $q$ is given by a tree as above. We then first check that if $q$ is a homogeneous polynomial of degree $\sigma$ in $\mathscr{A}^{\nu_{1}} \otimes \cdots \otimes \mathscr{A}^{\nu_{n}}$, then

$$
\begin{gathered}
\operatorname{TF}^{k} q(\xi)=\left(\xi_{1}+\cdots+\xi_{n}\right)^{k} T q(\xi), \\
\mathfrak{I} F^{k} q(\mathbf{j})=(-1)^{k}\left(\sigma-j_{1}-\cdots-j_{n}\right)_{k} \mathfrak{I} q(\mathbf{j}), \\
\mathbf{T} F^{k} q(\xi)=(-1)^{k}\left(\frac{1}{2}\left(\nu_{1}+\cdots+\nu_{n}\right)+\sigma-i\left(\xi_{1}+\cdots+\xi_{n}\right)\right)_{k} \mathbf{T} q(\xi) .
\end{gathered}
$$

Also $T(p \otimes q)=T p \otimes T q$, and similarly for $\mathfrak{I}$ and $\mathbf{T}$. If $q_{1}$ is a highest weight polynomial of degree $\sigma_{1}$ in $\mathscr{A}^{\nu_{1}} \otimes \cdots \otimes \mathscr{A}^{\nu_{m}}$ and $q_{2}$ one of degree $\sigma_{2}$ in $\mathscr{A}^{\nu_{m+1}} \otimes \cdots \otimes \mathscr{A}^{\nu_{n}}$, it then follows from (40) that 


$$
\begin{aligned}
& T\left(q_{1}, q_{2}\right)_{s} \\
= & \sum_{k=0}^{s}\left(\begin{array}{l}
s \\
k
\end{array}\right) \frac{(-1)^{s-k}\left(\mu_{1}\right)_{s}\left(\mu_{2}\right)_{s}}{\left(\mu_{1}\right)_{k}\left(\mu_{2}\right)_{s-k}}\left(\xi_{1}+\cdots+\xi_{m}\right)^{k}\left(\xi_{m+1}+\cdots+\xi_{n}\right)^{s-k} T q_{1} \otimes T q_{2},
\end{aligned}
$$

where $\mu_{1}=\nu_{1}+\cdots+\nu_{m}+2 \sigma_{1}$ and $\mu_{2}=\nu_{m+1}+\cdots+\nu_{n}+2 \sigma_{2}$. When $q$ is a highest weight polynomial given by a tree, this gives a product formula for $T q$ with one factor for each bifurcation point, each being of the form (with different $\mu_{1}, \mu_{2}, s, m$ and $n$ )

$$
\begin{aligned}
& \sum_{k=0}^{s}\left(\begin{array}{l}
s \\
k
\end{array}\right) \frac{(-1)^{s-k}\left(\mu_{1}\right)_{s}\left(\mu_{2}\right)_{s}}{\left(\mu_{1}\right)_{k}\left(\mu_{2}\right)_{s-k}}\left(\xi_{1}+\cdots+\xi_{m}\right)^{k}\left(\xi_{m}+1+\cdots+\xi_{n}\right)^{s-k} \\
& \left.=s !\left(-\xi_{1}-\cdots-\xi_{n}\right)^{s} P_{s}^{\left(\mu_{1}-1, \mu_{2}-1\right.}\right)\left(1-2 \frac{\xi_{1}+\cdots+\xi_{m}}{\xi_{1}+\cdots+\xi_{n}}\right),
\end{aligned}
$$

where $P$ is a Jacobi polynomial. For the operator $\mathfrak{I}$ we have

$$
\mathfrak{I}\left(q_{1}, q_{2}\right)_{s}(\mathbf{j})=\sum_{k=0}^{s}\left(\begin{array}{l}
s \\
k
\end{array}\right)(-1)^{k} \frac{\left(\mu_{1}\right)_{s}\left(\mu_{2}\right)_{s}\left(\sigma_{1}-\left|\mathbf{j}^{\prime}\right|\right)_{k}\left(\sigma_{2}-\left|\mathbf{j}^{\prime \prime}\right|\right)_{s-k}}{\left(\mu_{1}\right)_{k}\left(\mu_{2}\right)_{s-k}} \mathfrak{I} q_{1}\left(\mathbf{j}^{\prime}\right) \mathfrak{I} q_{2}\left(\mathbf{j}^{\prime \prime}\right),
$$

where $\mathbf{j}=\left(j_{1}, \ldots, j_{n}\right), \mathbf{j}^{\prime}=\left(j_{1}, \ldots, j_{m}\right)$ and $\mathbf{j}^{\prime \prime}=\left(j_{m+1}, \ldots, j_{n}\right)$, which gives a product formula for $\mathfrak{I} q$, each factor being of the form

$$
\begin{aligned}
& \sum_{k=0}^{s}\left(\begin{array}{l}
s \\
k
\end{array}\right)(-1)^{k} \frac{\left(\mu_{1}\right)_{s}\left(\mu_{2}\right)_{s}\left(\sigma_{1}-\left|\mathbf{j}^{\prime}\right|\right)_{k}\left(\sigma_{2}-\left|\mathbf{j}^{\prime \prime}\right|\right)_{s-k}}{\left(\mu_{1}\right)_{k}\left(\mu_{2}\right)_{s-k}} \\
& =(-1)^{s}\left(\mu_{2}\right)_{s}\left(\sigma_{1}+\sigma_{2}-|\mathbf{j}|\right)_{s} F_{2}\left(\begin{array}{c}
-s, \mu_{1}+\mu_{2}+s-1, \sigma_{2}-\left|\mathbf{j}^{\prime \prime}\right| \\
\mu_{2}, \sigma_{1}+\sigma_{2}-|\mathbf{j}|
\end{array} \mid 1\right) \\
& =(-1)^{s} s ! h_{s}^{\left(\mu_{1}-1, \mu_{2}-1\right)}\left(\left|\mathbf{j}^{\prime \prime}\right|-\sigma_{2},|\mathbf{j}|-\sigma_{1}-\sigma_{2}+1\right),
\end{aligned}
$$

where the latter two expressions are immediately verified by comparison with the identities leading to (38). Similarly there is a product formula for $\mathbf{T} q$, each factor being a Hahn polynomial of imaginary argument.

We will not write down these product formulas explicitly. Let us note, however, that we have found some special cases in the literature. In the case $n=3$ (when there is essentially only one tree), the polynomials $T q$ were introduced by Proriol in [25]. These have been applied to genetics and atomic physics, see the survey [16] for references. For general $n$ and for the special type of tree exemplified by Figures $1 \mathrm{~b}$ and $2 \mathrm{~b}$, the polynomials $\mathfrak{I} q$ are studied by Karlin and McGregor in [13], again in the context of genetics.

The product formula for $\mathfrak{I} q$ is of particular interest because we can use it to expand $q$ in monomials and the polynomials $T q$, I $q$ and $\mathbf{T} q$ in the gen- 
eralized Appell polynomials of the previous section. Indeed, if $\mathbf{s}$ and $\mathbf{t}$ are two multi-indices of the same length, the definition of $\mathfrak{I}$ gives

$$
\mathfrak{I z}^{\mathbf{s}}(\mathbf{t})= \begin{cases}0 & \mathbf{s} \neq \mathbf{t}, \\ \frac{s_{1} ! \cdots s_{n} !}{\left(\nu_{1}\right)_{s_{1}} \cdots\left(\nu_{n}\right)_{s_{n}}} & \mathbf{s}=\mathbf{t} .\end{cases}
$$

It follows that for any homogeneous polynomial $q$ of degree $s$,

$$
q(\mathbf{z})=\sum_{s_{1}+\cdots+s_{n}=s} \frac{\left(\nu_{1}\right)_{s_{1}} \cdots\left(\nu_{n}\right)_{s_{n}}}{s_{1} ! \cdots s_{n} !} \mathfrak{T} q\left(s_{1}, \ldots, s_{n}\right) z_{1}^{s_{1}} \cdots z_{n}^{s_{n}} .
$$

Using (9) we find

$$
\begin{aligned}
q(\mathbf{z})= & \sum_{s_{1}+\cdots+s_{n-1}=s} \frac{\left(\nu_{1}\right)_{s_{1}} \cdots\left(\nu_{n-1}\right)_{s_{n-1}}}{s_{1} ! \cdots s_{n-1} !} \mathfrak{I} q\left(s_{1}, \ldots, s_{n-1}, 0\right) \\
& \left(z_{1}-z_{n}\right)^{s_{1}} \cdots\left(z_{n-1}-z_{n}\right)^{s_{n-1}}
\end{aligned}
$$

and analogous expansions in $\prod_{j \neq i}\left(z_{j}-z_{i}\right)^{s_{j}}(i=1, \ldots, n-1)$. The results of the previous section then gives $n$ expressions for each of $T q, \mathfrak{I} q$ and $\mathbf{T} q$. Consequently each tree gives us a set of expansion formulas for orthogonal polynomials. As an example, let us write down some of these relations for $q=\left(\left(1_{\nu_{1}}, 1_{\nu_{2}}\right)_{s}, 1_{\nu_{3}}\right)_{t}$. The product formula for $\mathfrak{I} q$ is then, by (45),

$$
\begin{gathered}
\mathfrak{I} q\left(j_{1}, j_{2}, j_{3}\right)=(-1)^{s+t}\left(\nu_{2}\right)_{s}\left(\nu_{3}\right)_{t}\left(-j_{1}-j_{2}\right)_{s}\left(s-j_{1}-j_{2}-j_{3}\right)_{t} \\
\times_{3} F_{2}\left(\begin{array}{c}
-s, \nu_{1}+\nu_{2}+s-1,-j_{2} \\
\nu_{2},-j_{1}-j_{2}
\end{array} \mid 1\right){ }_{3} F_{2}\left(\begin{array}{c}
-t, \nu_{1}+\nu_{2}+\nu_{3}+2 s+t-1,-j_{3} \\
\nu_{3}, s-j_{1}-j_{2}-j_{3}
\end{array} \mid 1\right) .
\end{gathered}
$$

We now plug this into (46), replacing $s_{i}$ by $j_{i}$. Then $j_{1}+j_{2}+j_{3}=s+t$, so we can write

$$
\begin{gathered}
\left(s-j_{1}-j_{2}-j_{3}\right)_{t}=(-1)^{t} t ! \\
\left(-j_{1}-j_{2}\right)_{s}=\frac{(-1)^{s+j_{3}}\left(j_{1}+j_{2}\right) !(-t)_{j_{3}}}{t !}
\end{gathered}
$$

and (with $\left.\gamma=\nu_{1}+\nu_{2}+2 s+t-1\right)$

$$
{ }_{3} F_{2}\left(\begin{array}{c}
-t, \nu_{3}+\gamma,-j_{3} \\
\nu_{3}, s-j_{1}-j_{2}-j_{3}
\end{array} \mid 1\right)={ }_{2} F_{1}\left(\begin{array}{c}
\nu_{3}+\gamma,-j_{3} \mid \\
\nu_{3}
\end{array}\right)=\frac{(-\gamma)_{j_{3}}}{\left(\nu_{3}\right)_{j_{3}}},
$$

another instance of the Chu-Vandermonde formula. This gives 


$$
\begin{aligned}
& q(\mathbf{z})=\left(\nu_{2}\right)_{s}\left(\nu_{3}\right)_{t} \sum_{j_{1}+j_{2}+j_{3}=s+t} \frac{\left(j_{1}+j_{2}\right) !}{j_{1} ! j_{2} ! j_{3} !} \\
& \times\left(\nu_{1}\right)_{j_{1}}\left(\nu_{2}\right)_{j_{2}}(-t)_{j_{3}}\left(1-2 s-t-\nu_{1}-\nu_{2}\right)_{j_{3}} \\
& \times(-1)^{j_{3}}{ }_{3} F_{2}\left(\begin{array}{c}
\left.-s, \nu_{1}+\nu_{2}+s-1,-j_{2} \mid 1\right) z_{1}^{j_{1}} z_{2}^{j_{2}} z_{3}^{j_{3}} \\
\nu_{2},-j_{1}-j_{2}
\end{array}\right. \\
& =\left(\nu_{2}\right)_{s}\left(\nu_{3}\right)_{t} \sum_{j_{1}+j_{2}=s+t} \frac{(s+t) !\left(\nu_{1}\right)_{j_{1}}\left(\nu_{2}\right)_{j_{2}}}{j_{1} ! j_{2} !} \\
& \times{ }_{3} F_{2}\left(\begin{array}{c}
-s, \nu_{1}+\nu_{2}+s-1,-j_{2} \mid \\
\nu_{2},-s-t
\end{array}\right)\left(z_{1}-z_{3}\right)^{j_{1}}\left(z_{2}-z_{3}\right)^{j_{2}} \\
& =\left(\nu_{2}\right)_{s}\left(\nu_{3}\right)_{t} \sum_{j_{1}+j_{3}=s+t} \frac{\left(\nu_{1}\right)_{j_{1}}(-t)_{j_{3}}}{j_{3} !} \\
& \times\left(1-2 s-t-\nu_{1}-\nu_{2}\right)_{j_{3}}(-1)^{j_{3}}\left(z_{1}-z_{2}\right)^{j_{1}}\left(z_{3}-z_{2}\right)^{j_{3}}
\end{aligned}
$$

and one expansion in $\left(z_{2}-z_{1}\right)^{j_{2}}\left(z_{3}-z_{1}\right)^{j_{3}}$. (In this case these expansions are also easily obtained from Theorem 7.2. This seems not to be so for polynomials given by more complicated trees.) Proriol's polynomial $p\left(\xi_{1}, \xi_{2}\right)=(T q)\left(\xi_{1}, \xi_{2}, 1-\xi_{1}-\xi_{2}\right)$ can then be written, in terms of Jacobi polynomials $P$ and Appell's polynomials $\mathscr{F}$, as

$$
\begin{aligned}
p\left(\xi_{1}, \xi_{2}\right) & =(-1)^{s+t} s ! t !\left(\xi_{1}+\xi_{2}\right)^{s} P_{s}^{\left(\nu_{1}-1, \nu_{2}-1\right)}\left(1-2 \frac{\xi_{1}}{\xi_{1}+\xi_{2}}\right) \\
& \times P_{t}^{\left(\nu_{1}+\nu_{2}+2 s-1, \nu_{3}-1\right)}\left(1-2\left(\xi_{1}+\xi_{2}\right)\right) \\
& =\left(\nu_{2}\right)_{s}\left(\nu_{3}\right)_{t} \sum_{j_{1}+j_{2}=s+t} \frac{(s+t) !\left(\nu_{1}\right)_{j_{1}}\left(\nu_{2}\right)_{j_{2}}}{j_{1} ! j_{2} !}{ }_{3} F_{2}\left(\begin{array}{c}
-s, \nu_{1}+\nu_{2}+s-1,-j_{2} \\
\nu_{2},-s-t
\end{array}\right) \\
& \times \frac{(-1)^{s+t}}{\left(\nu_{3}\right)_{s+t}} \mathscr{F}_{j_{1}, j_{2}}\left(\nu_{1}+\nu_{2}+\nu_{3}-1, \nu_{1}, \nu_{2}, \xi_{1}, \xi_{2}\right)= \\
& \left(\nu_{2}\right)_{s}\left(\nu_{3}\right)_{t} \sum_{j_{1}+j_{3}=s+t} \frac{\left(\nu_{1}\right)_{j_{1}}(-t)_{j_{3}}\left(1-2 s-t-\nu_{1}-\nu_{2}\right)_{j_{3}}(-1)^{j_{3}}}{j_{3} !} \\
& \times \frac{(-1)^{s+t}}{\left(\nu_{2}\right)_{s+t}} \mathscr{F}_{j_{1} j_{3}}\left(\nu_{1}+\nu_{2}+\nu_{3}-1, \nu_{1}, \nu_{3}, \xi_{1}, 1-\xi_{1}-\xi_{2}\right) .
\end{aligned}
$$


The first of these expressions follows from (44), the other from (37) and (47). Applying $\mathfrak{I}$ and $\mathbf{T}$ leads to other identities involving orthogonal polynomials.

The matrix elements for a change between two of our bases (or rather the corresponding normalized bases) are the transformation coefficients, or Wigner symbols, of our representation. We conclude this section with a remark on the simplest non-trivial case, namely the Racah coefficients (or Wigner 6-j-symbols) corresponding to the bases (42). As far as we know, these are the only ones that can be expressed in terms of a single hypergeometric function, namely ${ }_{4} F_{3}(\mid 1)$ [31]. Our previous findings can be used to obtain a relatively simple deduction of this expression. This is based on the observation that the basis vectors can be expressed using Gauss' hypergeometric function ${ }_{2} F_{1}$. In fact, either from the last expression in (47) or from Theorem 7.2, we find, after some manipulations,

$$
\begin{aligned}
& \left(\left(1_{\nu_{1}}, 1_{\nu_{2}}\right)_{s}, 1_{\nu_{3}}\right)_{t}\left(z_{1}, z_{2}, z_{3}\right) \\
& =\left(\nu_{1}\right)_{s+t}\left(\nu_{2}\right)_{s}\left(\nu_{3}\right)_{t}\left(z_{1}-z_{2}\right)^{s+t}{ }_{2} F_{1}\left(\begin{array}{c|c}
-t, 1-2 s-t-\nu_{1}-\nu_{2} & \frac{z_{3}-z_{2}}{z_{1}-z_{2}}
\end{array}\right), \\
& \left(1_{\nu_{1}},\left(1_{\nu_{2}}, 1_{\nu_{3}}\right)_{u}\right)_{v}\left(z_{1}, z_{2}, z_{3}\right) \\
& =\left(\nu_{2}\right)_{u}\left(\nu_{3}\right)_{u}\left(\nu_{1}\right)_{v}\left(\nu_{2}+\nu_{3}+2 u\right)_{v}\left(z_{1}-z_{2}\right)^{v}\left(z_{2}-z_{3}\right)_{2}{ }_{2} F_{1}\left(\begin{array}{c|c}
-v, \nu_{3}+u \\
\nu_{2}+\nu_{3}+2 u
\end{array} \mid \frac{z_{3}-z_{2}}{z_{1}-z_{2}}\right) \text {. }
\end{aligned}
$$

If we now apply Chaundy's formula [6] (which is easily proved by elementary means):

$$
\begin{aligned}
& { }_{2} F_{1}\left(\begin{array}{c}
A, B \\
C
\end{array} \mid x\right) \\
& =\sum_{u=0}^{\infty} \frac{(a)_{u}(b)_{u}(-1)^{u}}{u !(c+u-1)_{u}} F_{3}\left(\begin{array}{c}
A, B, c+u-1,-u \mid \\
a, b, C
\end{array}\right) x_{2}^{u} F_{1}\left(\begin{array}{c}
a+u, b+u \mid x \\
c+2 u
\end{array}\right),
\end{aligned}
$$

with $A=-t, B=1-2 s-t-\nu_{1}-\nu_{2}$ and so on, we find after simplifications that

$$
\left(\left(1_{\nu_{1}}, 1_{\nu_{2}}\right)_{s}, 1_{\nu_{3}}\right)_{t}=\sum_{u+v=s+t} R_{s, t}^{u, v}\left(1_{\nu_{1}},\left(1_{\nu_{2}}, 1_{\nu_{3}}\right)_{u}\right)_{v}
$$

where 


$$
\begin{aligned}
& R_{s, t}^{u, v}=\frac{(-1)^{u} N !\left(\nu_{1}+v\right)_{u}\left(\nu_{2}\right)_{s}\left(\nu_{3}\right)_{t}\left(\nu_{2}+\nu_{3}+2 u-1\right)}{u ! v !\left(\nu_{2}\right)_{u}\left(\nu_{2}+\nu_{3}+u-1\right)_{N+1}} \\
& \times{ }_{4} F_{3}\left(\begin{array}{c}
\left.-t, 1-\nu_{1}-\nu_{2}-2 s-t, \nu_{2}+\nu_{3}+u-1,-\left.u\right|_{1}\right) \quad(s+t=u+v=N), \\
-N, \nu_{3}, 1-\nu_{1}-N
\end{array}\right.
\end{aligned}
$$

which thus, after normalization, are the Racah coefficients of our representation.

\section{Spherical harmonics.}

When the $\nu_{i}$ are all half-integers, the polynomials $p=T q$ of the previous two sections are related to spherical harmonics. In fact, if $q$ is a highest weight polynomial, then

$$
r\left(x_{1}, \ldots, x_{2|\nu|}\right)=(T q)\left(x_{1}^{2}+\cdots+x_{2 \nu_{1}}^{2}, \ldots, x_{2\left(\nu_{1}+\cdots+\nu_{n-1}\right)+1}^{2}+\cdots+x_{2|\nu|}^{2}\right)
$$

is harmonic, and if $q_{1}$ and $q_{2}$ are orthogonal in $\mathscr{A}^{\nu_{1}} \otimes \cdots \otimes \mathscr{A}^{\nu_{n}}$, then the corresponding polynomials $r_{1}$ and $r_{2}$ are orthogonal with respect to Euclidean measure on the unit sphere of $\mathrm{R}^{2|\nu|}$. This is not hard to prove directly: the harmonicity follows from the equations

$$
\sum_{i=1}^{2|\nu|} \frac{\partial^{2}}{\partial x_{i}^{2}} r=4 \sum_{i=1}^{n}\left(\xi_{i} \frac{\partial^{2}}{\partial \xi_{i}^{2}}+\nu_{i} \frac{\partial}{\partial \xi_{i}}\right) T q=4 T \sum_{i=1}^{n} \frac{\partial}{\partial z_{i}} q=0
$$

and the orthogonality is immediately reduced to (33).

We will now sketch an alternative approach to higher order Hankel forms based on the metaplectic representation, which gives a better understanding of these facts. This is in line with how similar problems are treated in [14]. We will assume that the $\nu_{i}$ are positive half-integers, so that $\mathscr{A}^{\nu_{i}}$ are representation spaces of the four-fold cover $G^{\prime}=\operatorname{Mp}(1)$ of $G$. We will consider the Segal-Shale-Weil (metaplectic, harmonic, oscillator) representation of $G^{\prime}$ on $L^{2}(\mathrm{R})$. Its $k$-th tensor power commutes with the natural action of $\mathrm{O}(k)$ on $\bigotimes^{k} L^{2}(\mathrm{R})=L^{2}\left(\mathrm{R}^{k}\right)$. (Actually, one can embed $G^{\prime}$ and $\mathrm{O}(k)$ as subgroups of $\mathrm{Mp}(k)$ in such a way that these two representations are obtained by restriction of the metaplectic representation of $\mathrm{Mp}(k)$. In the terminology of Howe [10], $\left(G^{\prime}, \mathrm{O}(k)\right)$ is then a dual pair.) The decomposition with respect to $G^{\prime} \times \mathrm{O}(k)$ is

$$
L^{2}\left(\mathrm{R}^{k}\right) \simeq \bigoplus_{s=0}^{\infty} \mathscr{A}^{\frac{k}{2}+s} \otimes \mathscr{H}_{s}^{k}
$$

where $\mathscr{H}_{s}^{k}$ is the irreducible representation of $\mathrm{O}(k)$ on homogeneous harmo 
nic polynomials in $k$ variables of degree $s$. In the case $k=1$ this is the finite decomposition

$$
L^{2}(\mathrm{R}) \simeq \mathscr{A}^{\frac{1}{2}} \oplus \mathscr{A}^{\frac{3}{2}}(=\text { even } \oplus \text { odd functions }) .
$$

If we view $\mathscr{A}^{\nu}$ as function spaces on the upper half-plane, the intertwining embeddings

$$
\mathscr{A}^{\frac{k}{2}+s} \otimes \mathscr{H}_{s}^{k} \rightarrow L^{2}\left(\mathrm{R}^{k}\right)
$$

are given by

$$
(g \otimes r)(x)=\hat{g}\left(\frac{|x|^{2}}{2}\right)|x|^{2-2\left(\frac{k}{2}+s\right)} r(x),
$$

where $\hat{g}$ is the Fourier transform of the boundary values of $g$ (cf. [31], Sections 7.7.8 and 12.3.2 for these facts).

Taking $s=0$ in (50), we may identify $\mathscr{A}^{\frac{k}{2}}$ with the space of rotation invariant functions in $L^{2}\left(\mathrm{R}^{k}\right)$, and thus $\bigotimes_{i=1}^{n} \mathscr{A}^{\frac{k_{i}}{2}}$ with the subspace of

$$
L^{2}\left(\mathrm{R}^{k}\right)=L^{2}\left(\mathrm{R}^{k_{1}}\right) \otimes \cdots \otimes L^{2}\left(\mathrm{R}^{k_{n}}\right), \quad k=\sum k_{i},
$$

invariant with respect to the subgroup

$$
\mathrm{O}\left(k_{1}\right) \times \cdots \times \mathrm{O}\left(k_{n}\right) \subseteq \mathrm{O}(k) .
$$

The decomposition (49) then gives

$$
\bigotimes_{i=1}^{n} \mathscr{A}^{\frac{k_{i}}{2}} \simeq \bigoplus_{s=0}^{\infty} \mathscr{A}^{\frac{k}{2}+s} \otimes \tilde{\mathscr{H}}_{s}^{k}
$$

where $\tilde{\mathscr{H}}_{s}^{k}$ is the subspace of $\mathscr{H}_{s}^{k}$ consisting of harmonic polynomials of the form

$$
r(x)=p\left(\left|x_{1}\right|^{2}, \ldots,\left|x_{n}\right|^{2}\right), \quad x_{i} \in \mathrm{R}^{k_{i}} .
$$

Let $V_{\frac{1}{2}}$ se the corresponding space of polynomials $p$. It is not hard to see that

$$
\operatorname{dim} V_{\frac{1}{2} s}= \begin{cases}0 & s \text { odd } \\
\left(\begin{array}{c}
n+\frac{1}{2} s-2 \\
n-2
\end{array}\right) & s \text { even, }\end{cases}
$$

which gives the decompositions

$$
\bigotimes_{i=1}^{n} \mathscr{A}^{\frac{k_{i}}{2}} \simeq \bigoplus_{s=0}^{\infty} \mathscr{A}^{\frac{k}{2}+2 s} \otimes V_{s}=\bigoplus_{s=0}^{\infty}\left(\begin{array}{c}
n+s-2 \\
n-2
\end{array}\right) \mathscr{A}^{\frac{k}{2}+2 s}
$$


with respect to $G^{\prime} \times \mathrm{O}(k)$ and $G^{\prime}$ respectively. So we recover (8) (in the case when the $\nu_{i}$ are half-integers).

From (51) one obtains explicit formulas for higher order Hankel forms. In fact, $g \otimes p$ corresponds to

$$
\begin{aligned}
& H\left(f_{1}, \ldots, f_{n}\right)=\int_{\mathrm{R}^{k}} \hat{f}_{1}\left(\frac{\left|x_{1}\right|^{2}}{2}\right)\left|x_{1}\right|^{2-k_{1}} \cdots \hat{f}_{n}\left(\frac{\left|x_{n}\right|^{2}}{2}\right)\left|x_{n}\right|^{2-k_{n}} \\
& \times|x|^{2-k-4 s} \bar{g}\left(\frac{|x|^{2}}{2}\right) p\left(\left|x_{1}\right|^{2}, \ldots,\left|x_{n}\right|^{2}\right) d x \\
& =C \int_{\mathrm{R}_{+}^{n}} \hat{f}_{1}\left(\xi_{1}\right) \cdots \hat{f}_{n}\left(\xi_{n}\right) \overline{\hat{b}\left(\xi_{1}+\cdots+\xi_{n}\right) p(\xi)} d \xi,
\end{aligned}
$$

where $\hat{b}(t)=t^{1-2 s-\frac{k}{2}} \hat{g}(t)$, so, as expected, we recover (31).

We also remark that it would lead to nothing new if we were to consider embeddings corresponding to $s>0$ in (50). This would, for any choice of $r_{i} \in \mathscr{H}_{t_{i}}^{k_{i}}(1 \leq i \leq n)$, allow us to identify $\bigotimes_{i=1}^{n} \mathscr{A}^{\frac{k_{i}}{2}+t_{i}}$ with functions in $L^{2}\left(\mathrm{R}^{k}\right)$ of the form

$$
r_{1}\left(x_{1}\right) \cdots r_{n}\left(x_{n}\right) F\left(\left|x_{1}\right|, \ldots,\left|x_{n}\right|\right), \quad x_{i} \in \mathrm{R}^{k_{i}},
$$

and thus give

$$
\bigotimes_{i=1}^{n} \mathscr{A}^{\frac{k_{i}}{2}+t_{i}} \simeq \bigoplus_{s=0}^{\infty} \mathscr{A}^{\frac{k}{2}+s} \otimes \tilde{\mathscr{H}}_{s}^{k},
$$

where now elements of $\tilde{\mathscr{H}}_{s}^{k}$ are harmonic polynomials of the form

$$
r(x)=r_{1}\left(x_{1}\right) \cdots r_{n}\left(x_{n}\right) p\left(\left|x_{1}\right|^{2}, \ldots,\left|x_{n}\right|^{2}\right), \quad x_{i} \in \mathrm{R}^{k_{i}} .
$$

However, the harmonicity of $r$ is equivalent to the harmonicity of the polynomial

$$
p\left(\left|x_{1}\right|^{2}, \ldots,\left|x_{n}\right|^{2}\right), \quad x_{i} \in \mathrm{R}^{k_{i}+2 t_{i}},
$$

so we recover the same classes $V_{s}$ and the same decomposition as above.

In [30] (cf. also [17, 31]) bases of the spaces $V_{s}$ indexed by labeled binary trees are constructed. Each tree then corresponds to a system of polyspherical coordinates, and the bases are obtained by separation of variables with respect to these. It is not hard to see that this is equivalent to what we did in the previous section, with the correspondence (48) between highest weight polynomials $q$ and harmonic polynomials $r$. This yields a proof of a fact first found in [15], namely that the Wigner symbols for the discrete series 
of $\mathrm{Mp}(1)$ agree with the "T-coefficients" corresponding to a change between polyspherical coordinates.

\section{REFERENCES}

1. P. Appell, Sur des polynômes de deux variables analogues aux polynômes de Jacobi, Arch. Math. Phys. 66 (1881), 238-245.

2. P. Appell, J. Kampé de Fériet, Fonctions hypergéométriques et hypersphériques. Polynomes d'Hermite, Gauthier-Villars, Paris, 1926.

3. J. Arazy and S. D. Fisher, Some aspects of the minimal, Möbius-invariant space of analytic functions on the unit disc, in M. Cwikel and J. Peetre, eds., Interpolation Spaces and Allied Topics in Analysis, 24-44, Lecture Notes in Math. 1070, (1984).

4. J. Arazy and S. D. Fisher, Invariant Hilbert spaces of analytic functions on bounded symmetric domains, in L. de Branges et al., eds., Topics in Operator Theory. Ernst D. Hellinger Memorial Volume, Operator Theory: Advances and Applications 48, Birkhäuser Verlag, Basel, 1990.

5. L. Biedenharn and J. Louck, The Racah-Wigner Algebra in Quantum Theory, AddisonWesley Publishing Company, Reading Mass., 1981.

6. T. W. Chaundy, Expansions of hypergeometric functions, Quart. J. Math., Oxford Ser. 13 (1942), 159-171.

7. F. Cobos, T. Kühn and J. Peetre, Schatten-von Neumann classes of multilinear forms, Duke Math. J. 65 (1992), 121-156.

8. R. Coifman and R. Rochberg, Representation theorems for holomorphic and harmonic functions in $L^{p}$, Astérisque 77 (1980), 11-66.

9. A. E. Djrbashian and F. A. Shamoian, Topics in the Theory of $A_{\alpha}^{p}$ Spaces, Teubner Verlagsgesellschaft, Leipzig, 1988.

10. R. Howe, Remarks on classical invariant theory, Trans. Amer. Math. Soc. 313 (1989), 539570 .

11. R. Howe and E. C. Tan, Non-Abelian Harmonic Analysis, Springer-Verlag, New York, 1992.

12. S. Janson and J. Peetre, A new generalization of Hankel operators (the case of higher weights), Math. Nachr. 132 (1987), 313-328.

13. S. Karlin and J. McGregor, Linear growth models with many types and multidimensional Hahn polynomials, in R. Askey, ed., Theory and Application of Special Functions, 261288, Academic Press, New York, 1975.

14. M. Kashiwara, M. Vergne, On the Segal-Shale-Weil representation and harmonic polynomials, Invent. Math. 44, 1-47 (1978).

15. V. A. Knyr, P. P. Pipirăte and Yu. F. Smirnov, Canonical transformations, "trees" and angular momenta which are multiples of 1/4, Sov. J. Nucl. Phys. 22 (1975), 554-558. (Russian original in Yad. Fiz. 22 (1975), 1063-1072.)

16. T. Koornwinder, Two-variable analogues of the classical orthogonal polynomials, in R. Askey, ed., Theory and Application of Special Functions, 435-495, Academic Press, New York, 1975.

17. A. F. Nikiforov, S. K. Suslov and V. B. Ubarov, Classical Orthogonal Polynomials of a Discrete Variable, Springer-Verlag, Berlin - Heidelberg, 1991.

18. J. Peetre, Invariant function spaces connected with the holomorphic discrete series, in $\mathrm{P}$. L. Butzer et al., eds., Anniversary Volume on Approximation Theory and Functional Analysis, 119-134, Birkhäuser Verlag, Basel, 1984.

19. J. Peetre, Orthogonal polynomials arising in connection with Hankel forms of higher weight, Bull. Sci. Math. (2) 116 (1992), 265-284.

20. J. Peetre, Hankel kernels of higher weight for the ball, Nagoya Math. J. 130 (1993), 183-192. 
21. J. Peetre, Hankel forms of arbitrary weight on bounded symmetric domains via the transvectant, Rocky Mountain J. Math. 24 (1994), 106-1085.

22. J. Peetre, Hankel forms of higher weight and classical invariant theory - an essay, in preparation.

23. J. Peetre and R. Rochberg, Higher order Hankel forms, Contemp. Math. 185 (1995), 283306.

24. M. Peloso, Möbius invariant spaces on the unit ball, Michigan Math. J. 39 (1992), 509-536.

25. J. Proriol, Sur une famille de polynomes à deux variables orthogonaux dans un triangle, C. R. Acad. Sci. Paris 245 (1957), 2459-2461.

26. L. Pukanszky, The Plancherel formula for the universal covering group of SL(2, R), Math. Ann. 156 (1964), 96-143.

27. H. Rosengren, Multivariable orthogonal polynomials arising as coupling coefficients for quantum algebra representations, in preparation.

28. P. Sally, Analytic continuation of the irreducible unitary representations of the universal covering group of SL(2, R), Mem. Amer. Math. Soc. 69 (1967).

29. H. M. Srivastava and P. W. Karlsson, Multiple Gaussian Hypergeometric Series, Ellis Horwood, Chichester, 1985.

30. N. Ja. Vilenkin, Polisfericheskie i orisfericheskie funktsii, Mat. Sb. 68 (1965), 432-443.

31. N. Ja. Vilenkin and A. U. Klimyk, Representation of Lie Groups and Special Functions, Vols. 1-3, Kluwer Academic Publishers, Dordrecht, 1991, 1992, 1993.

32. Z. Wu, Boundedness, compactness, and Schatten p-classes of Hankel operators between weighted Dirichlet spaces, Ark. Mat. 31 (1993), 395-417.

33. G. Zhang, Tensor products of weighted Bergman spaces and invariant Ha-plitz operators, Math. Scand. 71 (1992), 85-95.

DEPARTMENT OF MATHEMATICS

UNIVERSITY OF LUND

BOX 118

S-22100 LUND

SWEDEN

E-mail address: hjalmar@maths.lth.se 